\title{
The XMM large scale structure survey: optical vs. X-ray classifications of active galactic nuclei and the unified scheme ${ }^{\star}$
}

\author{
O. Garcet ${ }^{1}$, P. Gandhi ${ }^{2}$, E. Gosset ${ }^{1}$, P. G. Sprimont ${ }^{1}$, J. Surdej ${ }^{1}$, V. Borkowski ${ }^{1}$, M. Tajer ${ }^{3,4}$, F. Pacaud ${ }^{5,6}$, M. Pierre ${ }^{5}$,
} L. Chiappetti ${ }^{7}$, D. Maccagni ${ }^{7}$, M. J. Page ${ }^{8}$, F. J. Carrera ${ }^{9}$, J. A. Tedds ${ }^{10}$, S. Mateos ${ }^{10}$, M. Krumpe ${ }^{11}$, T. Contini ${ }^{12}$, A. Corral ${ }^{9}$, J. Ebrero ${ }^{9}$, I. Gavignaud ${ }^{11}$, A. Schwope ${ }^{11}$, O. Le Fèvre ${ }^{13}$, M. Polletta ${ }^{14}$, S. Rosen ${ }^{8}$, C. Lonsdale $^{14}$, M. Watson ${ }^{10}$, W. Borczyk ${ }^{15}$, and P. Vaisanen ${ }^{16}$

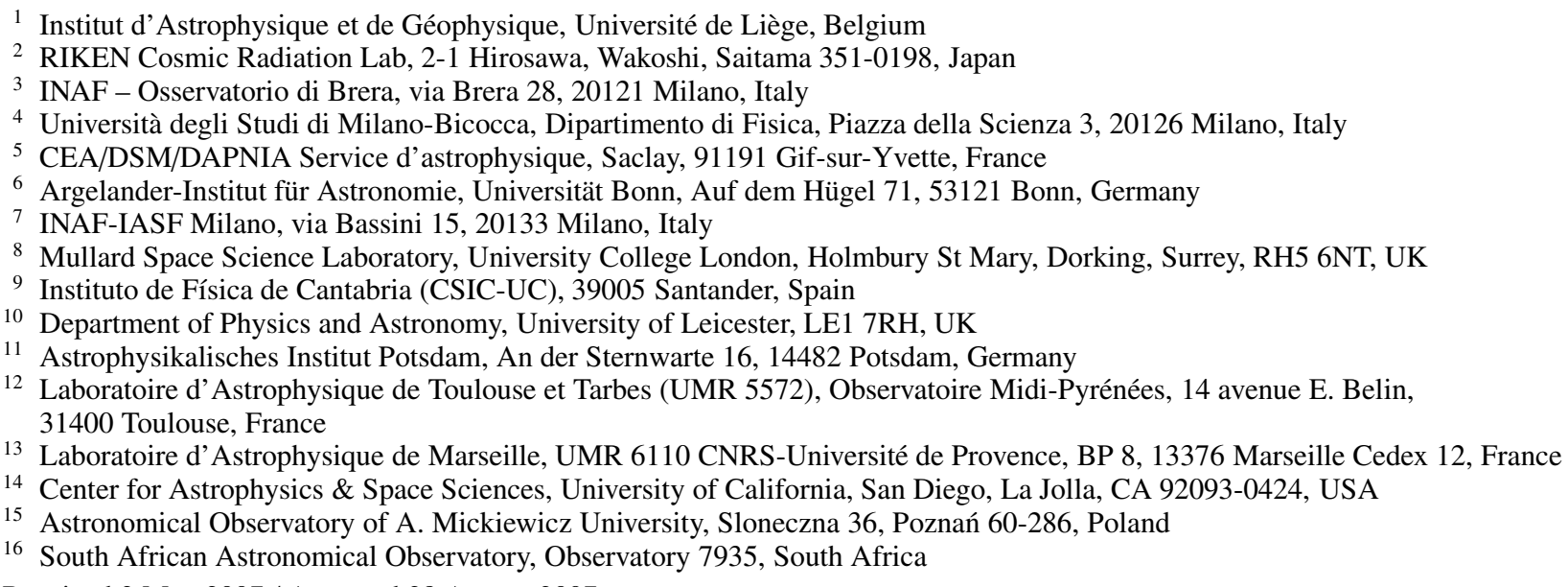

Received 2 May 2007 / Accepted 28 August 2007

\section{ABSTRACT}

\begin{abstract}
Aims. Our goal is to characterize AGN populations by comparing their X-ray and optical classifications within the framework of the standard orientation-based unified scheme.

Methods. We present a sample of 99 spectroscopically identified $(R \leq 22 \mathrm{mag})$ X-ray selected point sources in the XMM-LSS survey which are significantly detected $(\geq 3 \sigma)$ in the $[2-10] \mathrm{keV}$ band with fluxes between $8 \times 10^{-15}$ and $8 \times 10^{-14} \mathrm{erg} \mathrm{s}^{-1} \mathrm{~cm}^{-2}$, and which have more than 80 counts. We have compared their X-ray and optical classifications. To this end, we performed an X-ray spectral analysis for all of these $99 \mathrm{X}$-ray sources in order to assess whether they are intrinsically absorbed or not. The X-ray classification is based on the measured intrinsic column density. The optical classification is based on the measured $F W H M$ of the permitted emission lines, the absence of broad lines being due to obscuration within the framework of the standard AGN unified scheme.

Results. Introducing the fourfold point correlation coefficient $r$, we find a mild correlation between the X-ray and the optical classifications $(r=0.28)$, as up to $32 \mathrm{X}$-ray sources out of 99 have differing X-ray and optical classifications: on one hand, $10 \%$ of the type 1 sources $(7 / 32)$ present broad emission lines in their optical spectra and strong absorption $\left(N_{\mathrm{H}}\right.$ int $\left.\geq 10^{22} \mathrm{~cm}^{-2}\right)$ in the X-rays. These objects are highly luminous AGN lying at high redshift and thus dilution effects by the host galaxy light are totally ruled out, their discrepant nature being an intrinsic property instead. Their X-ray luminosities and redshifts distributions are consistent with those of the unabsorbed X-ray sources with broad emission lines $\left(L_{2-10} \sim 4 \times 10^{44} \mathrm{erg} \mathrm{s}^{-1} ; z \sim 1.9\right)$. On the other hand, $25 / 32$ are moderate luminosity $\left(L_{2-10} \leq 5 \times 10^{43} \mathrm{erg} \mathrm{s}^{-1}\right)$ AGN, which are both unabsorbed in the X-rays and only present narrow emission lines in their optical spectra. Based on their line ratios in the optical, the majority of them have an optical spectrum which is more representative of the host galaxy rather than of a reddened AGN. We finally infer that dilution of the AGN by the host galaxy seems to account for their nature. 5/25 have been defined as Seyfert 2 based on their optical spectra. In conclusion, most of these 32 discrepant cases can be accounted for by the standard AGN unified scheme, as its predictions are not met for only $12 \%$ of the 99 X-ray sources.
\end{abstract}

Key words. X-ray: galaxies - galaxies: active - surveys - galaxies: quasars: emission lines

\section{Introduction}

More than 40 years after its discovery, the X-ray background (XRB) is partially resolved into discrete sources and its main component is widely interpreted as being mostly made of AGN (Setti \& Woltjer 1989; Giacconi et al. 2002; Alexander et al. 2003). Recently, around $80 \%$ of the XRB has been

* Table 4 and Figs. A.1-A.3 are only available in electronic form at http: //wWw . aanda. org resolved in the [2-10] keV energy range by deep Chandra and XMM-Newton observations (e.g. Worsley et al. 2005; Hickox \& Markevitch 2006; Carrera et al. 2007). XRB synthesis models with a combination of absorbed and unabsorbed AGN and founded on the AGN orientation-based unified scheme (Antonucci 1993) in which each AGN contains an obscuring torus, have been able to reproduce the overall broadband spectral shape of the observed XRB (e.g. Comastri et al. 1995, 2001; Gandhi \& Fabian 2003; Treister \& Urry 2005; Gilli et al. 2007). 
The standard orientation-based unification scheme rather suggests that absorbing material (the putative torus) is present or not along the line of sight depending on the relative inclination of the torus. However, a certain number of observations do not agree with the predictions of these synthesis models (in particular with the unified scheme). There is a significant number of AGN for which the expected optical and X-ray characteristics are not the same, and are thus violating the unified scheme. On one hand, there are AGN showing strong absorption in the $\mathrm{X}$-rays while their optical and ultraviolet emission shows mild extinction (Fiore et al. 1999; Maiolino et al. 2001a; Page et al. 2001; Gallagher et al. 2006). On the other hand, there are also AGN which are only showing narrow emission lines in their optical spectra (which would happen e.g. if the broad line region was hidden), while only mild (or even absent) absorption is observed in their X-ray spectra (e.g. Pappa et al. 2001; Panessa \& Bassani 2002; Barcons et al. 2003). Finally, very recently, Punsly (2006) has used a sample including both obscured quasars and broad absorption line quasars (BAL QSOs) to compare their hydrogen column density derived from X-ray observations. They showed that, surprisingly, the BAL QSOs have column densities that are significantly larger than those of the obscured QSOs, which is at odds with the AGN unified scheme. In this paper, we characterize AGN populations over the XMM-LSS area by comparing their X-ray and optical classifications.

The XMM Large Scale Structure Survey (XMM-LSS) presently consists of 19 guaranteed-time (G) and 32 guestobserver time (B) overlapping pointings covering a total area of $6 \mathrm{deg}^{2}$. The nominal exposure times were $20 \mathrm{ks}$ and $10 \mathrm{ks}$ for the $\mathrm{G}$ and B pointings, respectively. We refer to Pierre et al. (2004) and Pierre et al. (2007) for details on the X-ray observations. Details of the detection pipeline and source classification are presented in Pacaud et al. (2006). The $19 \mathrm{G}$ pointings are part of the XMDS (XMM Medium Deep Survey, Chiappetti et al. 2005), which covers a total area of $3 \mathrm{deg}^{2}$ and which lies at the heart of the XMM-LSS. About two thirds of the XMDS area are covered in the optical band with the VVDS (VIRMOS VLT Deep Survey) both by UBVRI photometry (Le Fèvre et al. 2004) and by multi-object spectroscopy with VIMOS (Le Fèvre et al. 2005) and by an associated radio survey at $1.4 \mathrm{GHz}$ (Bondi et al. 2003). Part of the VVDS area $\left(\sim 0.8 \mathrm{deg}^{2}\right)$ is also covered by the UKIRT Infrared Deep Sky Survey (UKIDSS, Dye et al. 2006; Lawrence et al. 2007). Finally a large area of the XMM-LSS Survey has been covered by $2 \mathrm{dF}$ observations in December 2003 as part of the $11 \mathrm{deg}^{2}$ medium sensitivity XMM Wide Angle Serendipitous Survey (XWAS), using the $2 \mathrm{dF}$ optical multi fibre spectroscope on the AAT (Tedds et al., in prep.).

The present paper gathers a large and representative sample of 99 spectroscopically identified optical counterparts of X-ray point sources selected in the [2-10] keV band. The goal of our paper is to perform an internal comparison of the X-ray properties of our sample with the properties of their optical counterparts. Firstly, even if our sample is affected by selection effects (mostly concerning the optical counterparts), it will be shown that the conclusions of our work are not significantly affected by these incompleteness issues. Our results will finally be compared with the predictions of the standard AGN unified scheme (Antonucci 1993). Our work is a complementary analysis to the works of Tajer et al. (2007) and Polletta et al. (2007). They have selected a sample of $136 \mathrm{X}$-ray point sources detected at $\geq 3 \sigma$ in the [2-10] $\mathrm{keV}$ band within a $1 \mathrm{deg}^{2}$ area of the XMDS. Their goal was to probe the populations of AGN by a fit of SED templates over their optical+MIR photometric data points in order to infer the nature of the X-ray sources and their photometric redshifts. They only performed an X-ray spectral analysis for 55 sources, as most of them do not have enough counts.

The advantage of our study resides in that optical spectra with secure identification and redshift are available for each of the optical counterparts of the 99 point-like X-ray sources, and second, our sample covers a much larger area, about 3 times larger than the sample of Tajer et al. (2007), with about twice as many extracted X-ray spectra. This allows us to draw some stronger and safer statistical trends.

The sky area over which we have selected the AGN candidates has a very large overlap with the $4.2 \mathrm{deg}^{2}$ used by Gandhi et al. (2006) for an angular clustering analysis of AGN. While Gandhi et al. (2006) have discussed the clustering properties of sub-samples of absorbed and unabsorbed AGN separately, only minimal source identification and classification criteria were adopted by them. Our current paper is a big step towards fully classifying their moderately bright sample of AGN candidates and eventually studying the full three-dimensional clustering of AGN over a large sky area.

Our paper is organized as follows: the X-ray sample and the optical spectra are presented in Sect. 2. The optical classification is discussed in Sect. 3. The X-ray data reduction and the X-ray spectral analysis are presented in Sect. 4. In Sect. 5, we compare and discuss the optical obscuration and the X-ray absorption within the framework of the AGN unified scheme. In Sect. 6, we discuss the nature of the X-ray Bright Optically Normal Galaxies, while in Sect. 7, we present 7 type 2 QSO candidates. Finally, Sect. 8 provides a summary of the reported results. Throughout this paper, we assume $H_{0}=70 \mathrm{~km} \mathrm{~s}^{-1} \mathrm{Mpc}^{-1}$, $\Omega_{\mathrm{m}}=0.27$ and $\Omega_{\Lambda}=0.73$, in accordance with the WMAP cosmological parameters reported by Spergel et al. (2007).

\section{The samples}

\subsection{The sample of $X$-ray selected point-like sources}

We have used the most up-to-date X-ray catalog (Pierre et al. 2007 ) to define a sample of $612 \mathrm{X}$-ray selected point-like sources which have both a log-likelihood of detection $>20$ (this roughly corresponds to $>3 \sigma$ ) in the [2-10] keV band (see Pacaud et al. 2006, for full details), and a total number of counts $>80$ in the $[0.5-10] \mathrm{keV}$ band. These targets are located within the whole $6 \mathrm{deg}^{2}$ of the XMM-LSS, which have been observed so far. We used a minimum of 80 total counts in order to ensure sufficient photon-statistics to perform an X-ray spectral analysis. Figure A.1 in the appendix shows this sample of 612 X-ray sources, which are distributed among the $51 \mathrm{X}$-ray pointings.

As illustrated in the left panel of Fig. A.2 in the appendix, most of the $612 \mathrm{X}$-ray sources have a $2-10 \mathrm{keV}$ flux between $8 \times 10^{-15}$ and $8 \times 10^{-14} \mathrm{erg} \mathrm{s}^{-1} \mathrm{~cm}^{-2}$, and the turnover of the flux distribution is around $10^{-14} \mathrm{erg} \mathrm{s}^{-1} \mathrm{~cm}^{-2}$.

\subsection{The sample of $R \leq 22$ spectroscopically identified $X$-ray point sources}

Presently, optical spectra with secure identification and spectroscopic redshift are available for 99 X-ray sources out of 612. These $99 \mathrm{X}$-ray sources have been extracted in $26 \mathrm{X}$-ray pointings, and have a limiting flux of $8 \times 10^{-15} \mathrm{erg} \mathrm{s}^{-1} \mathrm{~cm}^{-2}$. Due to the fact that the X-ray pointings do overlap, the corresponding total area is smaller, around $3 \mathrm{deg}^{2}$.

In order to get the optical spectrum of the counterpart of each X-ray source, we have correlated our sample of 612 X-ray sources with the catalog of optical spectra observed so far, out 
to $5^{\prime \prime}$. Then, we only selected those X-ray sources which have an optical spectrum with a secure identification and a spectroscopic redshift. Proceeding this way, we end up with a sub sample of 99 X-ray sources. The histogram of the separations between the X-ray sources and the optical counterparts is shown in the left panel of Fig. A.3 in the appendix. Finally, we computed the probability that the association between an X-ray source and its putative optical counterpart results from random fluctuations using the following equation (Downes et al. 1986):

$p=1-\exp \left(-\pi n(<m) r^{2}\right)$,

where $r$ is the distance between the X-ray source and its optical counterpart, and $n(<m)$ is the density of objects brighter than the magnitude $m$ of the optical counterpart, which has been obtained by counting the number of sources brighter than a given $R$ magnitude based on the virphot catalog. This probability has been computed in order to check whether the optical spectrum is really the one of the X-ray source. As in Tajer et al. (2007), we ranked the probability that the association itself is non random as "good" ( $p<0.01)$, "fair" $(0.01<p<0.03)$ and "bad" ( $p>0.03)$. Out of the 94 optical counterparts for which the $R$ band magnitude is available, $94(100 \%)$ are classified as "good" ones. This is expected because most if not all of the optical counterparts are quite bright. This thus allows us to be highly confident in the proposed association between each X-ray source and its optical spectrum.

The optical spectra used in this work mainly issue from 3 spectroscopic runs: 79 of them have been taken with the $2 \mathrm{dF}$ in December 2003 (as part of the XWAS project) in two overlapping circular pointings, 2 degrees across and separated by 1.26 degrees. The pointing centers are: $02^{\mathrm{h}} 23^{\mathrm{m}} 52^{\mathrm{s}}-03^{\circ} 49^{\prime} 00^{\prime \prime}$ and $02^{\mathrm{h}} 25^{\mathrm{m}} 08^{\mathrm{s}}-05^{\circ} 02^{\prime} 27^{\prime \prime}$, respectively. The two pointings have been exposed for $4800 \mathrm{~s}$ and $3600 \mathrm{~s}$ respectively, both with the $300 \mathrm{~B}$ grating. The achieved spectral resolution of the $2 \mathrm{dF}$ spectra is around $R \sim 600$. The $\mathrm{S} / \mathrm{N}$ is about 5 at $5500 \AA$ for a source of magnitude $V=21$. Further details about the $2 \mathrm{dF}$ optical spectroscopy used in this work are provided in the XWAS catalogue paper (Tedds et al., in prep.). Note that 3 optical sources observed with $2 \mathrm{dF}$ have also been acquired independently with the Southern African Large Telescope (SALT) in 2006 with the PG0300 $(R \sim 400)$ and the PG0900 $(R \sim 1200)$ grisms during a PV phase. 9 spectra have been obtained with FORS2 at the VLT during follow-up campaigns of the XMM-LSS survey in 2002, 2003 and 2004. The combined exposure time is $1 \mathrm{~h} .8$ pointings have been exposed with the 600RI grism $(R \sim 1000)$ and one has been exposed with the $600 \mathrm{z}$ grism $(R \sim 1400)$. Finally, 11 spectra have been obtained with VIMOS at the VLT within the VVDS (Le Fèvre et al. 2005; Gavignaud et al. 2006). These objects were observed with the low-resolution red grism which covers the wavelength range 5500-9500 $\AA$ with a spectral resolution $R \sim 230$. The combined exposure time is $4.5 \mathrm{~h}$.

With this large number of spectroscopically identified X-ray sources, we are in a good position to start an analysis in which we characterize the population of X-ray sources in comparing their X-ray and optical properties.

Before starting this analysis, we have checked whether the sub sample of 99 X-ray sources constitutes a fair representation of the whole sample, which includes $612 \mathrm{X}$-ray sources. To this end, we first compared the [2-10] keV band flux distributions of these two samples. The histogram presented in the left panel of Fig. A.2 in the appendix shows the fraction of sources as a function of the [2-10] keV flux. It can be seen that the overall distributions of the flux for the sub sample of the 99 optically selected
X-ray point sources and for the 513 still optically unidentified $\mathrm{X}$-ray sources are in a rather good agreement with each other. Next, we also compared the hardness ratios for these same two samples. The hardness ratio is defined as:

$H R=\frac{H-S}{H+S}$

where $H$ is the background subtracted number of counts in the hard [2-10] keV band and $S$ is the background subtracted number of counts in the soft [0.5-2] keV band. The histograms of the hardness ratio for the two samples are presented in the right panel of Fig. A.2 in the appendix. This figure shows the fraction of X-ray sources in each bin for the hardness ratio. Once again, the two distributions are in good agreement with each other.

From both panels of Fig. A. 2 in the appendix, it can be seen that the 99 spectroscopically identified X-ray sources are slightly brighter and softer in the X-rays than the 513 still optically unidentified X-ray point sources.

Furthermore we have performed a Kolmogorov-Smirnov (K-S) test for both the hardness ratio and the flux distributions between the $99 \mathrm{X}$-ray sources which have an optical spectrum and the remaining $513 \mathrm{X}$-ray sources which do not have an optical spectrum, in order to test whether this trend is significant or not. We find that the probability that the two samples are drawn from the same population is $P=0.13$ and $P=0.15$, for the hardness ratio and the X-ray flux, respectively. Therefore, our sample of 99 optically identified X-ray point sources, for which the determining criterion to be included in the sample is the optical spectrum (which is not a uniform criterion at all) statistically constitutes a fair representation of the whole sample of the $612 \mathrm{X}$-ray sources. Optical selection effects will be discussed in the next section.

\section{The optical classification criteria}

To differentiate between type 1 and type 2 AGN, a value between $1000 \mathrm{~km} \mathrm{~s}^{-1}$ and $2000 \mathrm{~km} \mathrm{~s}^{-1}$ is often adopted for the relevant Full Width at Half Maximum (FWHM) border value of their corresponding emission lines (e.g. Page et al. 2006a; Caccianiga et al. 2004). In the present work, we did not use $1000 \mathrm{~km} \mathrm{~s}^{-1}$, as the $F W H M$ of some well known forbidden emission lines (e.g. [OII]) amounted up to $F W H M \sim 1100 \mathrm{~km} \mathrm{~s}^{-1}$. We decided to choose $1500 \mathrm{~km} \mathrm{~s}^{-1}$ as the dividing value to classify an AGN as either a type 1 or a type 2 object.

The FWHMs of all emission lines have been directly measured by fitting Gaussian profiles, for the 99 optical spectra, using the IRAF ${ }^{1}$ task splot from the package onedspec. We have checked that the spectral resolution of the optical spectra (the lowest being $R \sim 230$ ) allows us to distinguish a narrow emission line from a broad emission line, using $1500 \mathrm{~km} \mathrm{~s}^{-1}$ as the dividing value for the FWHM.

Following our convention, we have divided our sample into two optical classes:

\section{- Type 1 objects, including}

- 61 sources showing broad $\left(V_{F W H M} \geq 1500 \mathrm{~km} \mathrm{~s}^{-1}\right)$ permitted emission lines. These are Broad Emission Line AGN (BLAGN).

- Type 2 objects, including

- 35 sources showing narrow $\left(V_{F W H M}<1500 \mathrm{~km} \mathrm{~s}^{-1}\right)$ permitted emission lines. These are Narrow Emission Line Galaxies (NELG).

1 IRAF, which is an Image Reduction and Analysis Facility, is distributed by the National Optical Astronomy Observatories. 
Table 1. Optical spectroscopic classification.

\begin{tabular}{ccc}
\hline \hline Class & Spectral type & Number \\
\hline Type 1 & BLAGN & $\mathbf{6 1}$ \\
\hline Type 2 & NELG & 35 \\
& ALG & 3 \\
\hline & NELG+ALG & $\mathbf{3 8}$ \\
\hline Total & & $\mathbf{9 9}$ \\
\hline
\end{tabular}

- 3 sources showing no emission lines in their spectra. These are Absorption Line Galaxies (ALG).

These values are gathered in Table 1 . The fraction of type 1 (BLAGN) and type 2 objects (NELG+ALG) is thus $62 \%$ and $38 \%$ respectively.

Concerning the optical selection effects, we are biased against faint (typically $R>22$ ) optical counterparts, as can be seen in the right panel of Fig. A.3 in the appendix. In order to test whether the optical selection effects will significantly hamper the comparison between the X-ray and the optical properties, we searched for the fraction of similar types of AGN in much deeper surveys. Eckart et al. (2006), who have identified spectra of optical counterparts down to $R=24$, have found that $51 \%$ of their optical counterparts are BLAGN, which represents a fraction of BLAGN which is not significantly different from ours (within $2 \sigma$ ).

If we restrict their sample down to $R<22$, which is approximately the limiting magnitude of our analysis, they have identified $63 \%$ of BLAGN, which is very consistent with our corresponding fraction of BLAGN (within $1 \sigma$ ).

All this suggests that our internal comparison between the $\mathrm{X}$-ray and the optical properties does not suffer much from incompleteness issues, at least on statistical grounds.

Note that the fraction of BLAGN, NELG and ALG in our sample is also consistent with several other published studies (e.g. Fiore et al. 2003; Silverman et al. 2005). Della Ceca et al. (2004) have defined two samples: one in the [0.5-4.5] keV band, which is the XMM-Newton Bright Source Sample (BSS, hereafter) and the other one in the [4.5-7.5] keV band, which is the XMM-Newton Hard Bright Source sample (HBSS, hereafter). Concerning the BSS, they find a significantly higher fraction of broad line AGN (around 85\%). This can be explained by the fact that it corresponds to a band which is softer than our selected [2-10] keV band. In the HBSS, they find a fraction which is more consistent with ours (around 70\%, within $1 \sigma$ ).

The right panel of Fig. A.3 in the appendix shows our own $R$ band magnitude histogram of the 94 identified sources for which an $R$ band magnitude is available. The $R$ band magnitudes come from the UKST plate scans from the supercosmos archives in Edinburgh (equivalent to Cousins Vega magnitudes) and from the VVDS. Note that the lack of the $R$ band magnitude for the remaining 5 optical counterparts is solely due to partial optical imaging coverage.

Most of the sources have an $R$ band magnitude between 17 and 21 . We have mostly identified bright optical counterparts, due to the fact that most of our optical spectra come from the shallow $2 \mathrm{dF}$ run.

As shown in Fig. 1, type 1 AGN in our sample have been detected out to $z \sim 4$. These type 1 AGN have a broad distribution in redshift, most of them having $z<2.5$, which is consistent with the one observed by Tajer et al. (2007); see also Silverman et al. (2005). We have mostly identified type 2 AGN at $z<0.6$. The distribution of type $2 \mathrm{AGN}$ in our sample drops at a slightly lower redshift value than the one observed by Tajer et al. (2007).

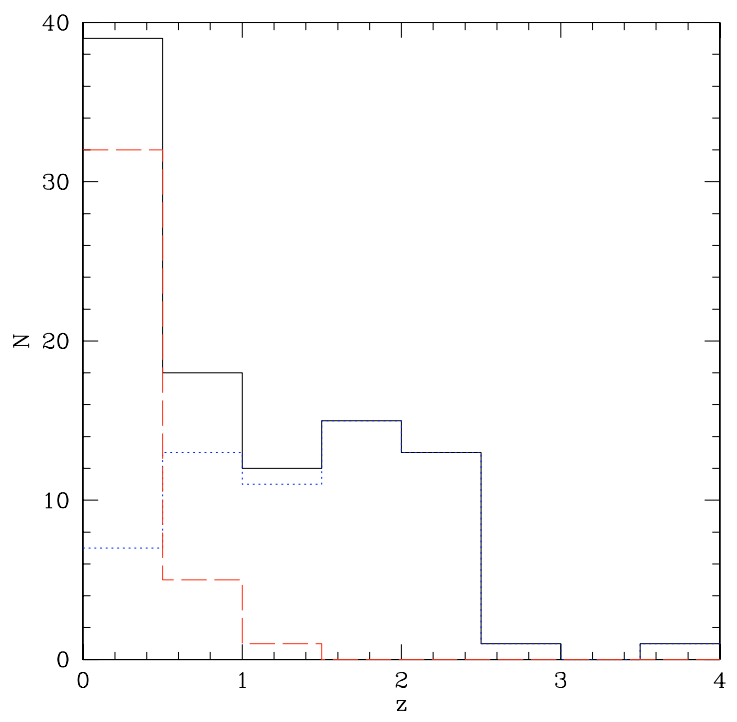

Fig. 1. Spectroscopic redshift distribution of the 99 optical counterparts in our sample. The solid histogram refers to the whole sample, the longdashed histogram refers to type $2 \mathrm{AGN}$ and the dotted histogram refers to type $1 \mathrm{AGN}$.

However this can mostly be accounted for by the fact that the bulk of our identified optical counterparts have a brighter optical magnitude. And thus statistically, they should lie at a lower redshift.

\section{X-ray spectral analysis}

\subsection{X-ray data reduction}

We have extracted the X-ray spectra for each of the 99 X-ray point sources of our sample, detected in the [2-10] keV band, for which we have an accurate redshift. Note that each X-ray source detected in the XMM-LSS survey has an off-axis distance smaller than 13', as the X-ray pipeline (Pacaud et al. 2006) detects X-ray sources with an off-axis distance up to $13^{\prime}$.

The X-ray data reduction has been performed using the most recent version of the XMM-Newton Science Analysis System (SAS v7.0.0). All valid event patterns (PATTERN 0-12) were used in constructing the MOS spectra. For the pn spectra, only the single and double events (PATTERN 0-4) have been used. Events were extracted for each source using the SAS task evselect in a circular region with a radius varying between $20^{\prime \prime}$ and $30^{\prime \prime}$, depending on the off-axis distance of the X-ray source. The background events have been extracted in the nearest source free circular region, on the same CCD chip, excluding areas near CCD gaps. We have tried several sizes for the background extraction region, between $20^{\prime \prime}$ and $60^{\prime \prime}$ : a small region is not really representative of the background underneath the source (noise problem) whereas a large region includes background counts too far away from the source where the background might be significantly different. Moreover, it would also increase the probability to include other X-ray sources in the background region. We ended up choosing a radius around $40^{\prime \prime}$ for the background extraction region as we found it to be a good compromise. The background regions have been chosen manually, in order to avoid to include other X-ray sources.

In order to perform a proper spectral analysis, we created the redistribution matrix file (RMF) and the ancillary response file (ARF) for each X-ray source and for each of the used detectors, using the SAS tasks rmfgen and arfgen, respectively. To make 
full use of the available X-ray data, we have simultaneously fitted the X-ray spectra of the 3 detectors (MOS1, MOS2, and pn), whenever possible.

For the cases consisting of X-ray sources detected over two $\mathrm{X}$-ray pointings, for which the off-axis distances are less than $12^{\prime}$ and the separation on the two X-ray pointings is less than $1^{\prime}$, we have jointly extracted their X-ray spectra, in order to keep a good PSF and to have rmf files that are close enough to each other. There were 5 such cases. For the other cases, we have chosen the X-ray pointing with the longest exposure time, while if the exposure times are equivalent, we have chosen the pointing for which the X-ray source has the smaller off-axis distance.

\subsection{Results}

The X-ray spectra have been analyzed using XSPEC (v11.3.0). We have kept the [0.3-10] keV region for spectral fitting and we ignored bad energy channels. We fitted as many of the 3 detectors as possible. We binned the spectra with at least 8 energy bins, in order to have enough energy bins to fit a powerlaw model to the X-ray spectra. When at least 15 counts are available in each energy bin, $\chi^{2}$ statistic was used. Otherwise, we used Cash statistic (Cash 1979), in which case we binned the spectra to have at least 5-10 counts in each energy bin. Among our 99 X-ray spectra, 41 of them have been fitted using $\chi^{2}$ statistic and 58 of them have been fitted using Cash statistic. When the $\chi^{2}$ statistic was applied, we verified that the Cash statistic yielded consistent results.

For each X-ray spectrum, we first fitted the spectrum using an absorbed powerlaw model with galactic hydrogen column density along the line of sight $\left(N_{\mathrm{H}}=2.6 \times 10^{20} \mathrm{~cm}^{-2}\right.$; Dickey \& Lockman 1990) and a possible intrinsic absorption component at the source redshift. This corresponds to the XSPEC model phabs*zphabs* pow, fixing the galactic column density to the value given above and using the spectroscopic redshift of the source. When $\chi^{2}$ statistic was used, we fitted the spectrum setting both the intrinsic column density $\left(N_{\mathrm{H}}^{\mathrm{int}}\right)$ and the photon index $(\Gamma)$ as free parameters. In cases where the fitted value of $N_{\mathrm{H}}^{\mathrm{int}}$ is consistent with 0 (at the $95 \%$ confidence level), we refitted the X-ray spectrum using the XSPEC model phabs*zpow, with $\Gamma$ as the only free parameter.

Otherwise, when Cash statistic was used, we never set both the $N_{\mathrm{H}}^{\mathrm{int}}$ and the $\Gamma$ as free parameters, as there were not sufficient counts to constrain both parameters. So, when using Cash statistic, the fit was first performed with $N_{\mathrm{H}}^{\text {int }}$ as a free parameter, fixing $\Gamma$ to a value of 1.9 , which is a value representative of broad line AGN (Turner \& Pounds 1989; Nandra \& Pounds 1994). Each time the inferred $N_{\mathrm{H}}^{\text {int }}$ was consistent with 0 (at the $95 \%$ confidence level), we refitted the spectra only taking into account the galactic absorption component (XSPEC model phabs*zpow) and with $\Gamma$ as the only free parameter. 7 sources out of 99 are better fitted (significant increase of the goodness of fit) when a more complicated model than a powerlaw is used: 1 needed an absorbed powerlaw+black body model, 1 two power laws, one absorbed and one unabsorbed with the same value for $\Gamma$, and for 5 sources, the presence of an emission line was required ( 2 have been tentatively identified as the $\mathrm{Fe} \mathrm{K}$ line at $6.4 \mathrm{keV}$, using the spectroscopic redshift of the source).

We have classified our X-ray sources using a discriminating value of $N_{\mathrm{H}}^{\text {int }}=10^{22} \mathrm{~cm}^{-2}$ for the best fit value of the intrinsic hydrogen column density. This value has been chosen as it corresponds to the column density of neutral hydrogen needed to hide the broad line regions for clouds which have a standard gas-to-dust ratio (Silverman et al. 2005).

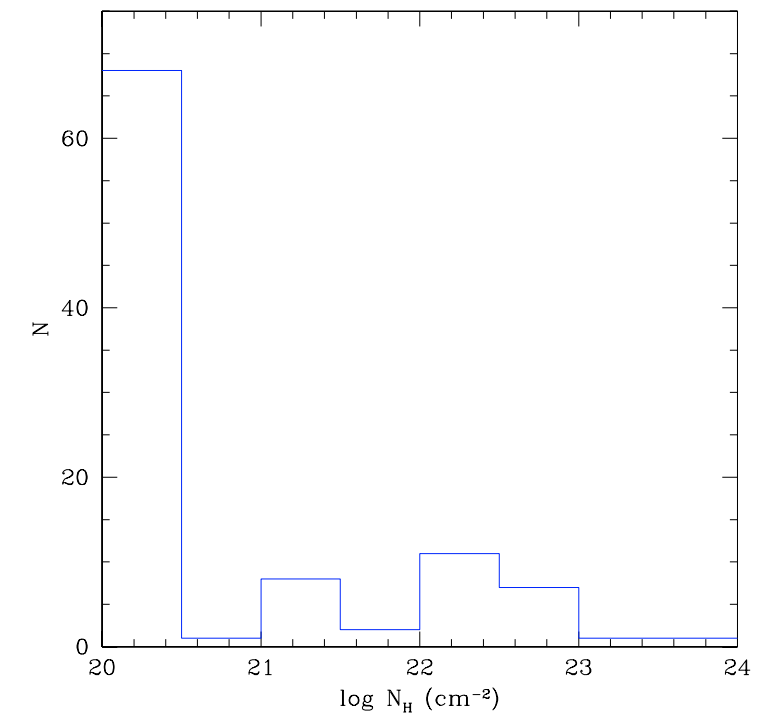

Fig. 2. Column density distribution (galactic+intrinsic component) for the whole sample.

Above this threshold, the AGN is considered as absorbed in the X-rays (hereafter type II). Otherwise, the AGN is unabsorbed (hereafter type I). Using this criterion we end up with 79 X-ray sources classified as type I AGN and 20 sources classified as type II AGN. Tajer et al. (2007) report a significantly higher fraction of type II AGN (49\%) but this is mostly due to our different selection method. Indeed, as our study is only keeping X-ray sources with more than 80 counts in the $[0.5-10] \mathrm{keV}$ band, it is likely that we identify mostly type I AGN.

Furthermore, as Tajer et al. (2007) use photometric redshifts, they go much deeper in magnitude (although with larger redshift uncertainties) for the optical photometric points over which a spectral energy distribution template is fitted in order to assess both the redshift and identify the nature of the X-ray source.

The observed frame X-ray flux, and the rest frame, deabsorbed (intrinsic) X-ray luminosity in the [2-10] keV band have been derived for the $99 \mathrm{X}$-ray sources, directly from the model fitted to the X-ray spectrum.

The distribution of $N_{\mathrm{H}}$ for our sample is shown in Fig. 2. The first bin in the histogram corresponds to the fixed galactic value for the hydrogen column density. This refers to X-ray spectra for which a power law model with no intrinsic absorption has been fitted, as we have checked that X-ray absorption is inconsistent at the $95 \%$ confidence range for these X-ray sources.

Concerning the type I X-ray sources we have made the histogram of the 60 fitted values of $\Gamma$ shown in Fig. 3. Our distribution of the fitted value for the photon index has a mean value of $\Gamma=2.01 \pm 0.28$, which is consistent with the works of Page et al. (2006a), Caccianiga et al. (2004), Mainieri et al. (2002, 2006), and Mateos et al. (2005).

\section{Optical obscuration versus $\mathrm{X}$-ray absorption}

In this section, we compare optical obscuration and X-ray absorption within the framework of the AGN unified scheme. The simple orientation-based unified model (Antonucci 1993) predicts a strict correlation between the optical obscuration and the $\mathrm{X}$-ray absorption. However, recently, quite a few studies have claimed to find objects for which the X-ray and the optical classifications do not match. For example, Panessa \& Bassani (2002) have presented a significant number of type 2 AGN whose X-ray 


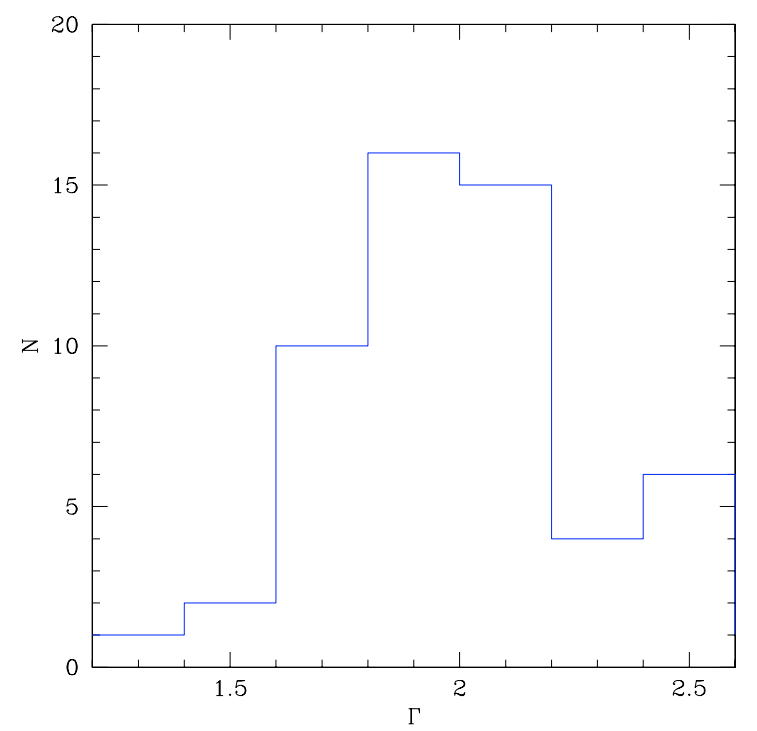

Fig. 3. Distribution of the photon indices that have been fitted for the $\mathrm{X}$-ray sources classified as type I.

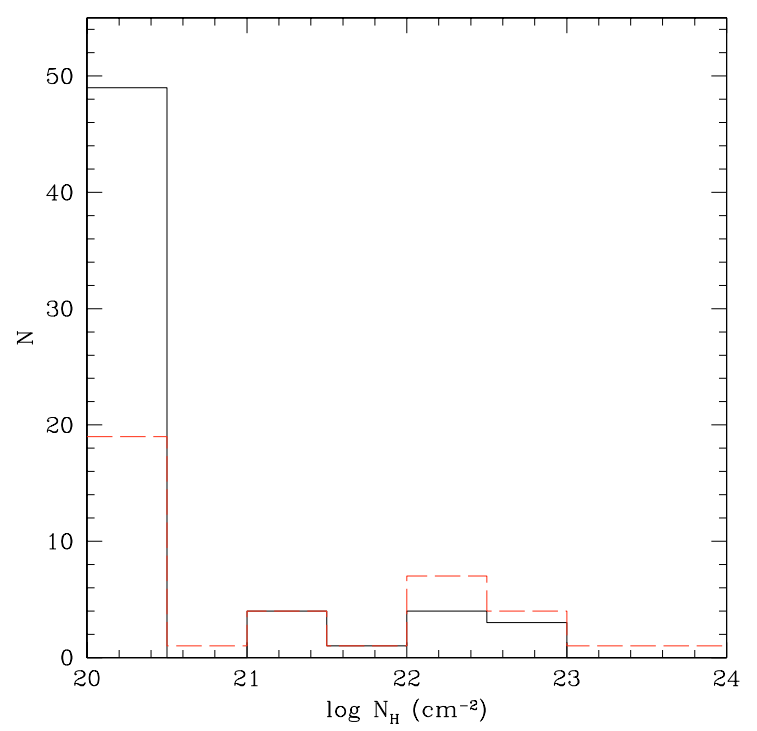

Fig. 4. Column density distribution (galactic+intrinsic component) for type 1 AGN (solid histogram) and for type 2 AGN (long dashed histogram).

spectra are indicative of a mild (or absent) absorption. They tentatively estimated this percentage in the range 10-30\%. Another important exception to the X-ray/optical classification correlation is the discovery of type 1 AGN with large X-ray absorption (e.g. Fiore et al. 1999), some of them being Compton thick (Gallagher et al. 2006). Finally, very recently, Page et al. (2006a) also reported a significant number of type 2 AGN with low X-ray absorption.

In the present work, we also find a significant number of $\mathrm{X}$-ray sources for which the X-ray and the optical classifications do not match. Figure 4 illustrates the column density for type 1 AGN (solid histogram) and for type 2 AGN (long dashed histogram). This figure shows that $20 \%$ of the type 1 objects have $N_{\mathrm{H}}>10^{21} \mathrm{~cm}^{-2}$ and $11 \%$ have $N_{\mathrm{H}}>10^{22} \mathrm{~cm}^{-2}$. Tajer et al. (2007) find that about $31 \%$ of their type 1 AGN show strong $\mathrm{X}$-ray absorption $\left(N_{\mathrm{H}}>10^{22} \mathrm{~cm}^{-2}\right)$. They inferred the intrinsic column density from a sample of X-ray spectra and from an
Table 2. Number of sources as a function of the optical (type 1 or type 2) and the X-ray (type I or type II) classifications.

\begin{tabular}{cccc}
\hline \hline & Type I & Type II & $N$ \\
\hline Type 1 & 54 & 7 & 61 \\
Type 2 & 25 & 13 & 38 \\
\hline N & 79 & 20 & $\mathbf{9 9}$ \\
\hline
\end{tabular}

analysis of the hardness ratios for the X-ray sources which do not have a sufficient number of counts to extract their X-ray spectra. Only taking into account the X-ray sources for which an X-ray spectrum has been extracted, they find a value much closer to ours, around $9 \%$ which is in good agrement.

On the other hand, $66 \%$ of our type 2 objects have $N_{\mathrm{H}}<$ $10^{22} \mathrm{~cm}^{-2}$ and $53 \%$ have $N_{\mathrm{H}}<10^{21} \mathrm{~cm}^{-2}$. This is consistent with the results of Page et al. (2006a) who find that about $68 \%$ of their type 2 AGN are showing very low absorption in the X-rays.

Table 2 shows the number of sources divided according to their X-ray and optical classifications. This table shows that there is a large probability that a BLAGN is a type I, as 54/61 $(89 \%)$ of them are unabsorbed in the X-rays. Also, there is a large probability that an absorbed AGN is a type 2, as 13/20 $(65 \%)$ of them only present narrow emission lines in their optical spectra. This trend has been previously observed many times (e.g. Mainieri et al. 2006; Page et al. 2006a; Tajer et al. 2007). However, in overall, the optical and the X-ray classifications are only matched for $68 \%$ of the AGN in our sample. At first sight, $68 \%$ still seems to be large. In order to test in more details this correlation, we thus computed the fourfold point correlation coefficient (hereafter $r$ ) which is defined as:

$r=\frac{n_{11} n_{22}-n_{12} n_{21}}{\sqrt{n_{1 .} n_{2 .} n_{.1} n_{.2}}}$

where $n_{i j}$ is the value of the element $i j$ of the table; $n_{1}$. corresponds to $n_{11}+n_{12} ; n_{2}$. corresponds to $n_{21}+n_{22} ; n_{.1}$ corresponds to $n_{11}+n_{21}$ and $n_{.2}$ corresponds to $n_{12}+n_{22}$. The fourfold point correlation coefficient is similar to the ordinary correlation coefficient: when this coefficient is equal to 1 , there is a strict correlation, when it is equal to 0 , there is no correlation at all, and finally when it is equal to -1 , there is an anti-correlation. We introduce this correlation coefficient because it corresponds to a more rigorous way to quantify the correlation between the X-ray and the optical classifications: if no correlation is observed between the X-ray and the optical classifications $(r=0)$, we would find $25 \%$ (in case of equipartition among the two types) of the objects in each box of Table 2, which would mean that the X-ray and the optical classification would be matched for up to $50 \%$ of the X-ray sources.

We thus applied Eq. (3) to the elements of Table 2 and found a value of $r=0.275_{-0.098}^{+0.132}$. We defined one-sided error bars, which have been computed assuming poisson errors according to Gehrels (1986) for low number statistic, on each of the number of sources $n_{i j}$ and using the error propagation equation starting from Eq. (3). We have tested these error bars in producing 1000000 Monte Carlo simulations assuming Poisson statistic in the presence of correlation. We find very similar error bars, even if slightly smaller. Finally, we have produced the same kind of simulation but in the presence of no correlation (i.e. $r=0$ ). The dispersion obtained around the value $r=0$ is 0.1 . Thus, the value of $r$ that we obtain for the whole sample $\left(r=0.275_{-0.098}^{+0.132}\right)$ is consistent with a mild correlation but is significantly $(2 \sigma$ level $)$ different from the case $r=0$. Note that the value of $r$ that we have 


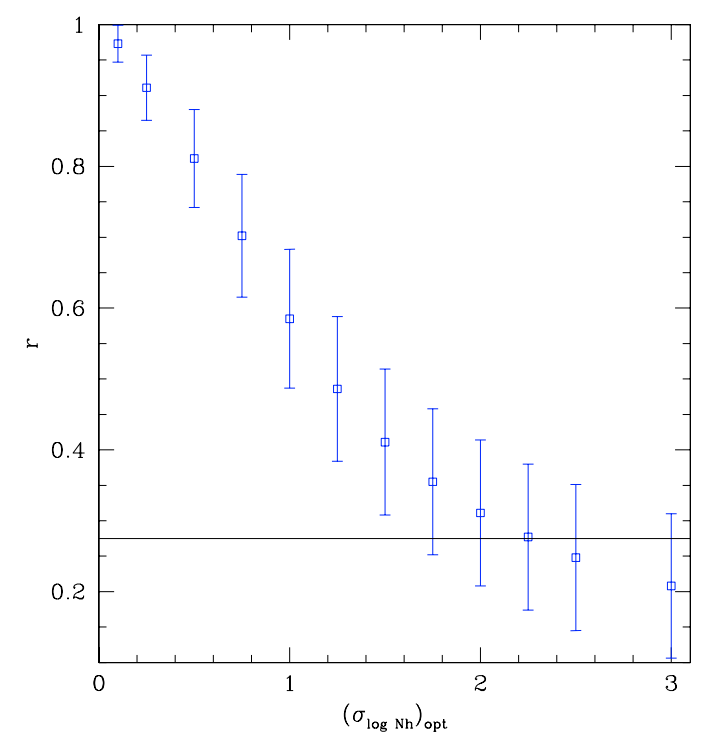

Fig. 5. The average value of the $r$ coefficient over 100000 simulations as a function of the standard deviation $\sigma$ of the assumed Gaussian distribution of the logarithm of the column density in the optical. The error bars for the $r$ value correspond to $1 \sigma$. The solid line corresponds to the value $r=0.275$ that we obtain for our sample of $99 \mathrm{X}$-ray sources.

obtained is totally inconsistent with a strict correlation between the X-ray and the optical classifications, even if the two classifications are matched for up to $68 \%$ of the X-ray sources in the sample, as a strict correlation between the X-ray and the optical classification would yield a value compatible with $r=1$.

In order to better clarify the meaning of the result $r=0.275$, we have produced 100000 samples of 99 X-ray sources for which we have computed the coefficient $r$, proceeding in the following way: in each simulated sample, we have assumed for the optical obscuring column in each source a value drawn from a Gaussian distribution around the $N_{\mathrm{H}}$ derived from the X-ray spectral analysis. We have then classified the X-ray sources using a threshold of $N_{\mathrm{H}}=10^{22} \mathrm{~cm}^{-2}$, both in the X-rays (actually no change occurs) and in the optical. Finally, we have computed the average value of the coefficient $r$ over the 100000 simulations, as a function of the standard deviation $\sigma$ of the Gaussian distribution of the logarithm of the column density in the optical. The results that we have obtained from these simulations are shown in Fig. 5. This Figure clearly indicates that a value of $r=0.275$ corresponds to a rather large width for the Gaussian distribution of the logarithm of the column density in the optical, which means that there is at most a mild correlation between the $\mathrm{X}$-ray and the optical classifications. More quantitatively, these simulations have shown that a value of $r=0.275$ is consistent with the $\sigma$ of the Gaussian distribution being equal to 2. In other words, it means that $32 \%$ of the X-ray sources have their column density in the X-rays and in the optical which differ by more than a factor 100 . In order to take into account the fact that X-ray sources with a column density fixed to the galactic value might have mild intrinsic X-ray absorption, we have obtained new simulations setting $N_{\mathrm{H}}=10^{21} \mathrm{~cm}^{-2}$ for these X-ray sources. With this value, we find that $32 \%$ of the X-ray sources have their column density in the X-rays and in the optical which differ by more than a factor 40 . Therefore, these simulations have clearly shown that there is at most a mild correlation between the X-ray and the optical absorption properties.

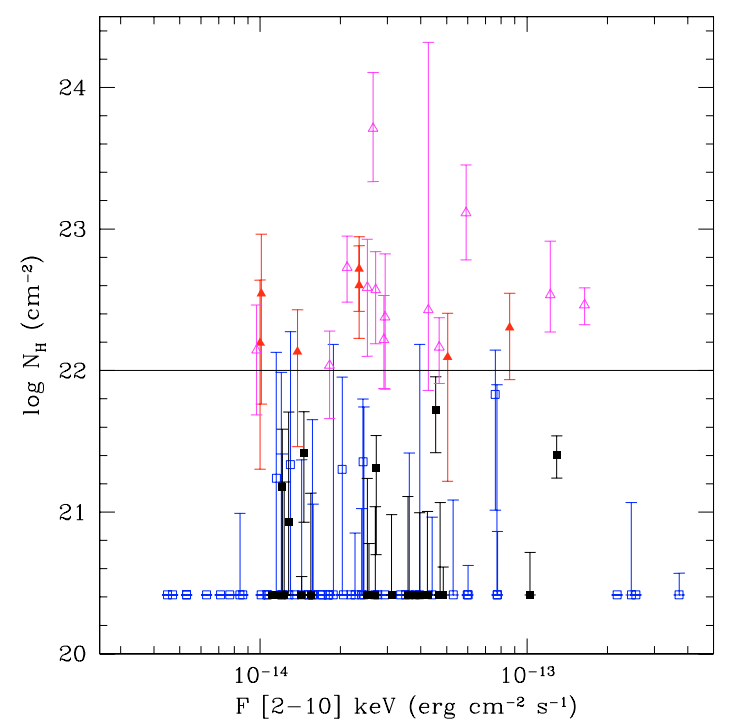

Fig. 6. Column density distribution (galactic+intrinsic component) as a function of the absorbed [2-10] keV flux for the $99 \mathrm{X}$-ray sources. Squares are X-ray sources classified as type I while triangles correspond to X-ray sources classified as type II. Filled symbols are the sources for which the X-ray and the optical classifications do not match. The error bars correspond to the $95 \%$ confidence interval. Data points without error bars correspond to sources for which the column density has been fixed to the galactic value. These column densities are consistent with the galactic value and the presence of absorption in the relevant X-ray spectra is at least rejected at the $95 \%$ level. The horizontal line corresponds to the dividing line between type I X-ray sources (for which $\left.N_{\mathrm{H}}<10^{22} \mathrm{~cm}^{-2}\right)$ and type II X-ray sources $\left(N_{\mathrm{H}}>10^{22} \mathrm{~cm}^{-2}\right)$.

As it will be shown later, sources \#44 and \#63 (both being unabsorbed X-ray sources, classified as type 2 AGN) are embedded in an X-ray galaxy cluster. So for these 2 sources, the difference between the X-ray and the optical classifications might be due to a soft contribution from the X-ray galaxy cluster. We have thus also computed $r$ excluding these two X-ray sources. We end up with $r=0.294_{-0.100}^{+0.134}$. Even if slightly higher, the conclusion remains unchanged.

When applied to the sample of Tajer et al. (2007), we find a value of $r=0.252_{-0.085}^{+0.106}$. Their result is consistent with ours within the error bars. Their value of $r$ is also consistent with the absence of a strict correlation between the X-ray and the optical classifications.

We then computed the fourfold point correlation coefficient $r$ as a function of the X-ray flux and the X-ray luminosity in the [2-10] keV band: we first defined 3 sub samples having a flux limit of 1,3 and $5 \times 10^{-14} \mathrm{erg} \mathrm{s}^{-1} \mathrm{~cm}^{-2}$. The $r$ values obtained are $0.228_{-0.103}^{+0.140}, 0.163_{-0.157}^{+0.252}$ and $0.433_{-0.220}^{+0.431}$, respectively.

We then also defined 3 sub samples having a luminosity larger than $10^{42} \mathrm{erg} \mathrm{s}^{-1}, 10^{43} \mathrm{erg} \mathrm{s}^{-1}$ and $10^{43.5} \mathrm{erg} \mathrm{s}^{-1}$. We end up with $r$ values of $0.285_{-0.102}^{+0.138}, 0.205_{-0.127}^{+0.191}$ and $0.261_{-0.166}^{+0.331}$, respectively.

Figure 6 shows the hydrogen column density as a function of the absorbed [2-10] keV band flux. This figure clearly shows that the fraction of sources for which the X-ray and the optical classifications do not match does not seem to increase significantly towards faint X-ray fluxes, i.e. there is no bias or selection effect in flux. Note that 16 X-ray sources out of 99 have their $95 \%$ confidence intervals crossing the discriminating line of $N_{\mathrm{H}}=10^{22} \mathrm{~cm}^{-2}(11 \mathrm{X}$-ray absorbed and $5 \mathrm{X}$-ray unabsorbed sources). In order to estimate their influence on the fourfold 
point correlation coefficient $r$, we switched their X-ray classifications (11 X-ray unabsorbed and $5 \mathrm{X}$-ray absorbed) and have computed the corresponding correlation coefficient $r$. We obtain a value of $r=0.097_{-0.097}^{+0.144}$. We have also computed the correlation coefficient excluding these $16 \mathrm{X}$-ray sources: $r=0.281_{-0.098}^{+0.161}$.

Therefore the influence of these 16 ambiguous X-ray sources does not significantly alter our results on the comparison between the X-ray and the optical properties, e.g., at most a mild correlation is observed.

Therefore, the mismatch we observe between the X-ray and the optical classifications is a general result which does not strongly depend on the X-ray flux or luminosity. Note that for a flux greater than $5 \times 10^{-14} \mathrm{erg} \mathrm{s}^{-1} \mathrm{~cm}^{-2}$ or a luminosity larger than $10^{43.5} \mathrm{erg} \mathrm{s}^{-1}$, this result is not as significant anymore. We obtain a value of $r=0.348_{-0.101}^{+0.151}$ when considering the sample of Page et al. (2006a), which is consistent with our result.

Silverman et al. (2005) find that $81 \%$ of their X-ray sources can be easily interpreted in the context of current AGN unification models. Proceeding the same way, we obtain a value of at most $r=0.58$ for their sample: it has to be considered as an upper limit: as most of their inferred column density are upper limits, we cannot tell whether the X-ray and the optical classifications are matched for those cases. Their value, even if larger, is still consistent with our results, within the error bars. Very recently, Mainieri et al. (2006) have presented a sample of $135 \mathrm{X}$-ray sources in the XMM-COSMOS field. From their data, we infer a coefficient $r=0.458_{-0.079}^{+0.103}$. Once more, this value of $r$ is consistent with only a mild correlation between the X-ray and the optical properties, in good agreement with our own results.

Finally, our results are significantly different from those of Caccianiga et al. (2004) who have defined a sample of 28 bright $\mathrm{X}$-ray sources with spectroscopic identifications: they find that the strict correlation predicted by the unified scheme is almost always respected: all their type 1 objects are only showing mild absorption in the X-rays. On the contrary, all but one of their type 2 are characterized by column densities larger than $10^{22} \mathrm{~cm}^{-2}$. So $96 \%$ of their X-ray sources have a similar classification in the $\mathrm{X}$-rays and the optical. We have computed the fourfold point correlation coefficient for their analysis. We find a value of $r=$ $0.91 \pm 0.26$, showing that there is a strong correlation $(r=1)$ between the X-ray and the optical classifications for the X-ray sources in their sample, which is totally consistent with the unified scheme. The significant differences with our sample are that their sample is solely composed of X-ray sources with very bright fluxes $\left(F_{2-10}>8 \times 10^{-14} \mathrm{erg} \mathrm{s}^{-1} \mathrm{~cm}^{-2}\right)$ and that their sample is flux limited in the [4.5-7.5] keV band.

If we restrict our sample to X-ray sources brighter than this flux limit, we only end up with 9 X-ray sources, for which the coefficient $r=0.316_{-0.282}^{+0.645}$. So, at these very bright $\mathrm{X}$-ray fluxes, our results are consistent within the error bars, with those of Caccianiga et al. (2004). However, this is mainly due to the lack of identification of very bright X-ray sources in our sample: only 9 X-ray sources out of 99 have $F_{2-10}>8 \times 10^{-14} \mathrm{erg} \mathrm{s}^{-1} \mathrm{~cm}^{-2}$. So, it would be very interesting to increase the size of our sample, especially towards very bright X-ray fluxes, in order to check whether the absence of a correlation between the X-ray and the optical classifications is still observed.

In Fig. 7, we have plotted the column density as a function of the $2-10 \mathrm{keV}$ intrinsic rest-frame luminosity. No correlation between the absorbing column and the intrinsic X-ray luminosity is found.

In this section, we have shown that, statistically, no strong correlation is observed between the X-ray and the optical properties. This result seems to contradict the predictions of the AGN

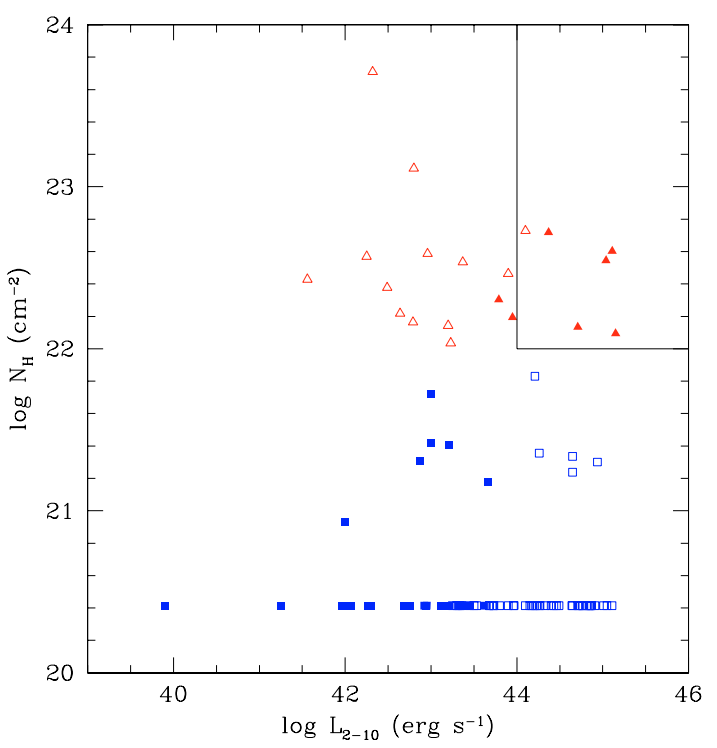

Fig. 7. Column density (galactic+intrinsic component) as a function of the $2-10 \mathrm{keV}$ intrinsic rest-frame luminosity. Squares are $\mathrm{X}$-ray sources classified as type I while triangles are those classified as type II. Filled symbols are the sources for which the X-ray and the optical classifications do not match. The solid lines mark the region where type II QSOs should be found.

unified scheme. Therefore, in the following two subsections, we will now discuss the physical nature of these $32 \mathrm{X}$-ray sources for which the X-ray and the optical classifications do not match, in order to investigate in further details whether the predictions of the standard unified scheme are really not met. We will discuss the 25 unabsorbed X-ray sources presenting only narrow emission lines in their optical spectra in Sect. 5.1, and the 7 absorbed X-ray sources presenting obvious and broad emission lines in their optical spectra, in Sect. 5.2.

\subsection{Unabsorbed AGN lacking broad emission lines in their optical spectra}

\subsubsection{Broad band properties}

Figure 8 shows a representative example of an unabsorbed X-ray source, which is classified as a type 2 . This X-ray source is showing mild absorption $\left(N_{\mathrm{H}}=2.5 \times 10^{21} \mathrm{~cm}^{-2}\right)$ with $\Gamma$ fixed to 1.9 . This is source \#53 in Table 4, which lists the X-ray and optical properties of all the $99 \mathrm{X}$-ray sources. We have found 25 such cases amongst our sample.

We have compared the distribution of the photon index, for the unabsorbed X-ray sources which are type 1, and type 2 (Fig. 9). The distribution of the photon index for the type 2 is significantly lower than the one for the type 1 . The distribution for the type 2 is peaking around $\Gamma \sim 1.9$.

As can be seen in Fig. 10, the vast majority of these discrepant objects (filled squares; 84\%) are lying below $z=0.5$. However they span a broad range of X-ray luminosities, from $L_{2-10}=10^{40} \mathrm{erg} \mathrm{s}^{-1}$ to almost $L_{2-10}=5 \times 10^{43} \mathrm{erg} \mathrm{s}^{-1}$.

As a comparison, only $11 \%$ of the type I X-ray sources which are presenting broad emission lines (empty squares) are lying below $z=0.5$, which is significantly less than for the type 2 . This result is partially due to optical selection effects, as we did not identify many type 2 AGN at high redshifts $(z \geq 1)$. However, it also means that unabsorbed X-ray bright AGN are less often found as the redshift $z$ decreases. 

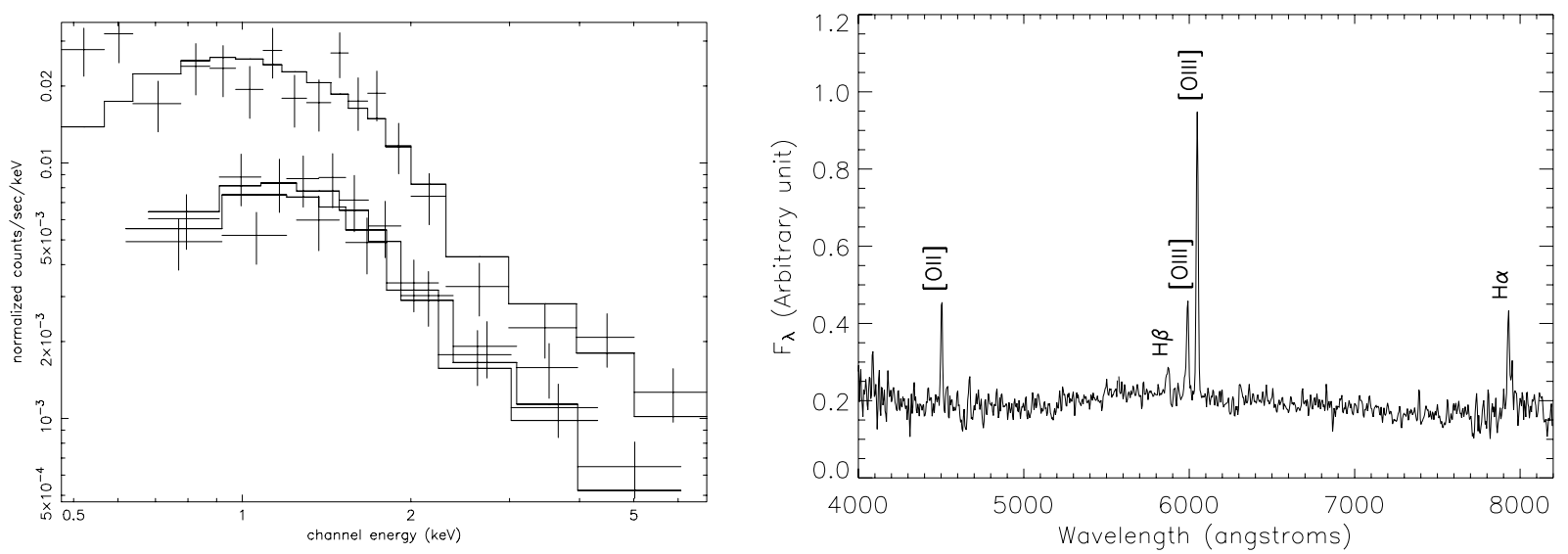

Fig. 8. MOS1 MOS2 (the two lower functions) and pn (upper one) X-ray spectra of a source which is only showing mild X-ray absorption with $N_{\mathrm{H}}^{\mathrm{int}}=2.5 \times 10^{21} \mathrm{~cm}^{-2}$. The crosses represent the data points and the solid lines represent the folded model simultaneously fitted to the data of the 3 detectors $(l e f t)$. The optical counterpart has been identified as a Seyfert 2 lying at a redshift $z=0.207$. We clearly observe the [OIII] lines around $6000 \AA$ (right). This is source \#53 in Table 4 .

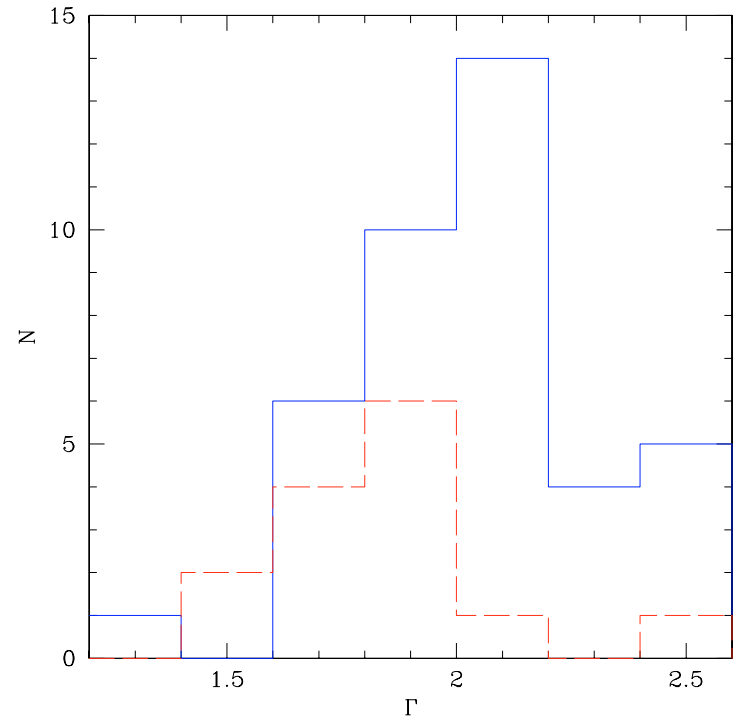

Fig. 9. Distribution of the photon index for type I X-ray sources which are classified as type 1 (solid histogram) and as type 2 (long dashed histogram).

For sources with $L_{2-10}<10^{42} \mathrm{erg} \mathrm{s}^{-1}$, the presence of an $\mathrm{AGN}$ is no longer certain. Actually these lower luminosity X-ray sources could be powered by very active star formation galaxies instead. In these objects, the X-rays may be partially due to the emission of X-ray binaries, supernovae, and/or to optically thin thermal emission from hot interstellar gas within the host galaxy. There are only 3 type I/type 2 sources (namely sources \#13, \#55 and \#63) with X-ray luminosity below $10^{42} \mathrm{erg} \mathrm{s}^{-1}$. One of them (source \#63) is embedded in a galaxy cluster detected in the X-rays, and so the difference in classification for the X-ray and the optical might be due to the soft emission from the cluster, which has a temperature around $0.6 \mathrm{keV}$ (see Pierre et al. 2006). These $3 \mathrm{X}$-ray sources with $L_{2-10}<10^{42} \mathrm{erg} \mathrm{s}^{-1}$ have an $\mathrm{X}$-ray/optical flux ratio well below 0.1 , as can be seen in Fig. 11 (the three filled squares). So a priori, they are more likely starbursts than AGN (Fiore et al. 2003).

Ranalli et al. (2003) have presented tight linear relations between the X-ray, radio and infrared luminosities of a welldefined sample of star-forming galaxies. We have used their

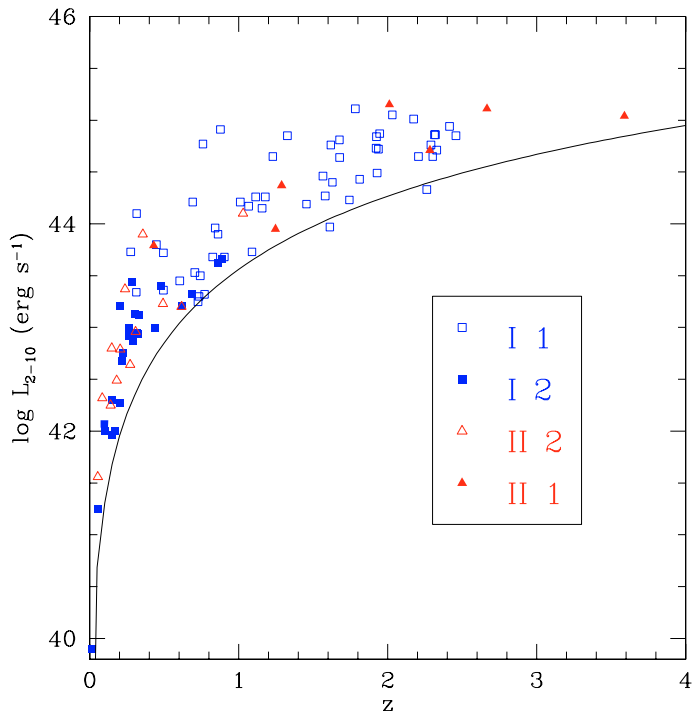

Fig. 10. 2-10 keV intrinsic rest-frame luminosities as a function of redshift for our sample of X-ray sources. Squares are X-ray sources classified as type I while triangles are those classified as type II. Filled symbols are the sources for which the X-ray and the optical classifications do not match. The solid line is showing the $2-10 \mathrm{keV}$ luminosity as a function of redshift for a $2-10 \mathrm{keV}$ limiting flux of $8 \times$ $10^{-15} \mathrm{erg} \mathrm{s}^{-1} \mathrm{~cm}^{-2}$.

relations in order to test the starburst hypothesis. We thus checked for radio emission using the radio catalogs from Cohen et al. (2003) and the one from Bondi et al. (2003), which is lying in the central $1 \mathrm{deg}^{2}$ area of the VVDS area. For these three starburst candidates, only one (source \#63) has a radio counterpart $(1 \mathrm{mJy}$ at $1.4 \mathrm{GHz})$. We infer a radio luminosity $L_{1.4 \mathrm{GHz}}=1.65 \times 10^{29} \mathrm{erg} \mathrm{s}^{-1} \mathrm{~Hz}^{-1}$ from its redshift $z=0.054$. With an X-ray luminosity of $L_{2-10} \sim 2 \times 10^{41} \mathrm{erg} \mathrm{s}^{-1}$, it does not follow the linear relation from Ranalli et al. (2003) (i.e. its $\mathrm{X}$-ray luminosity is about 8 times higher than inferred from its radio luminosity, which is much larger than expected for starforming galaxies, supporting the assumption that this source is an AGN). Using Fig. 13 of Polletta et al. (2007), source \#63 is neither on the star-forming galaxies nor on the radio-quiet correlations. It will be shown in Sect. 5.1.2 that this X-ray source 


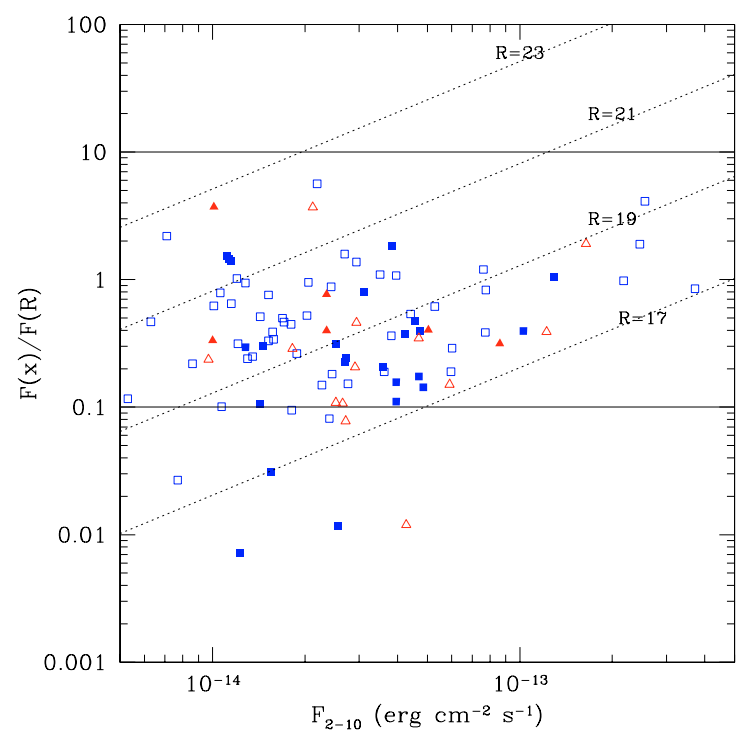

Fig. 11. X-ray to optical ratio ( $2-10 \mathrm{keV}$ band vs. $R$ band) as a function of X-ray flux for the $94 \mathrm{X}$-ray sources in our sample for which an $R$ band magnitude is available. Diagonal dotted lines indicate loci of constant $R$ magnitude while horizontal solid lines mark the boundary lines of the region of canonical AGN. The symbol convention is the same as for Fig. 10.

is in fact a Seyfert 2, based on emission line ratios in its optical spectrum.

Source \#13 has the lowest X-ray luminosity of our sample $\left(L_{2-10} \sim 10^{40} \mathrm{erg} \mathrm{s}^{-1}\right)$, along with a very low X-ray/optical flux ratio, typical of star-forming galaxies. Thus this X-ray source is more likely a starburst.

Source \#55 has an X-ray luminosity $L_{2-10} \sim 9 \times 10^{41} \mathrm{erg} \mathrm{s}^{-1}$, which is pretty close to the AGN threshold. So it could be either an AGN, or a star-forming galaxy.

For the remaining 22 objects with $L_{2-10}>10^{42} \mathrm{erg} \mathrm{s}^{-1}$, the starburst hypothesis is no longer valid, as the presence of an AGN is unambiguous. These $22 \mathrm{X}$-ray sources are thus likely AGN dominated objects. Furthermore 20/20 (only 20/22 have an $R$ magnitude) have an $\mathrm{X}$-ray/optical flux ratio between 0.1 and 10 (Fig. 11), which are typical values for AGN (Fiore et al. 2003). Note that source \#44 is also embedded in a galaxy cluster and is actually the cD galaxy of the cluster. For this source, the mismatched classification between the X-ray and the optical might also be due to additional soft emission from the galaxy cluster, its temperature being around $2 \mathrm{keV}$ (Pierre et al. 2006).

Moran et al. (2002) have obtained integrated (i.e. nucleus+host galaxy) optical spectra of a sample of nearby Seyfert 2 galaxies absorbed in the X-rays band. They find that, due to the limitations of optical spectroscopic observations, X-ray absorbed and optically type 2 AGN could be easily undetected in the optical band. They suggested that host galaxy dilution is a possible explanation for the lack of AGN lines in their sample of AGN optical spectra.

More recently, Severgnini et al. (2003) and Silverman et al. (2005) found a significant number of unabsorbed X-ray sources that only present narrow emission lines in their optical spectra. They argued that it could also be attributed to dilution of the AGN emission by the host galaxy light. In other words, in these AGN, the nuclear component will be outshone by the optical light of the host galaxy.

Page et al. (2006a) tested this hypothesis and found that for more than half of their obscured AGN in the optical, the nuclear

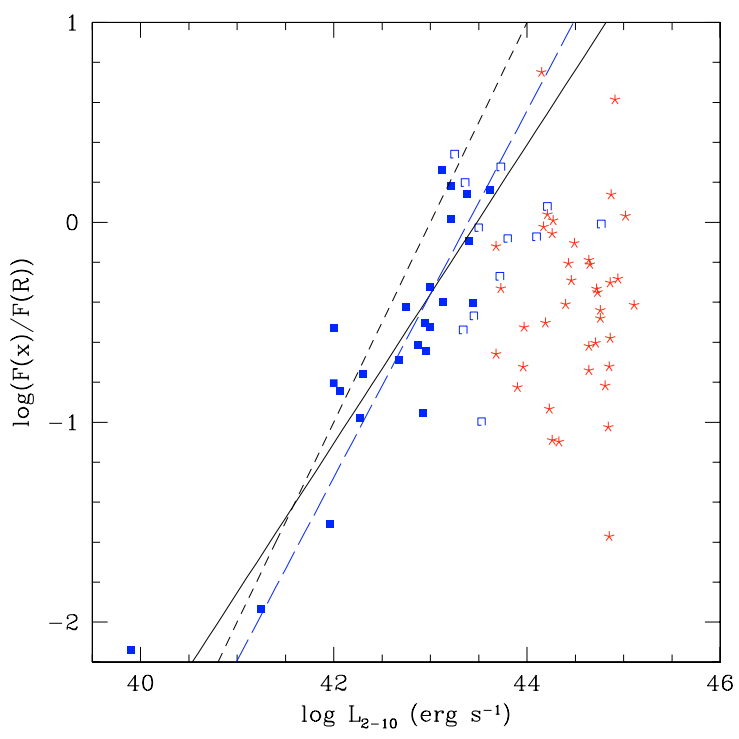

Fig. 12. X-ray to optical ratio $(2-10 \mathrm{keV}$ band vs. $R$ band) as a function of the deabsorbed, intrinsic $2-10 \mathrm{keV}$ luminosity for the type I objects. Some of them are only presenting narrow emission lines (filled squares). The type I that show broad and obvious emission lines in their optical spectra are divided into two categories: those which lie at $z<0.8$ (empty squares) and those at $z>0.8$ (stars). The solid line represents the minimum $\chi^{2}$ fit between $\log \left(F_{\mathrm{x}} / F_{\text {opt }}\right)$ and $\log L_{2-10}$ for our data. The short dashed line represents the best linear regression for type 2 AGN, obtained using the data of Fiore et al. (2003), and the long dashed line is from the data of Treister et al. (2005).

component is indeed outshone by the host galaxy by factors of 3-10. And so they claim that the lack of broad optical emission lines could be due to the low contrast of the emission lines against the much stronger starlight component of the host galaxy.

Concerning our $22 \mathrm{AGN}$, the dilution hypothesis is possible but not absolutely certain. As it can be seen in Fig. 11, there is a small trend for these objects (filled squares) to have both a brighter optical magnitude and a lower X-ray/optical flux ratio than the unabsorbed sources which are type 1 AGN (open squares), thus supporting the dilution hypothesis. However this trend is not very significant: a K-S test has shown that the $\mathrm{X}$-ray/optical flux ratio of these $22 \mathrm{AGN}$ is consistent with the one which are type 1 AGN $(P \sim 0.4)$, and that their optical $R$ magnitude is barely significantly brighter than for the type 1 AGN $(P \sim 0.07)$. Therefore, this diagram alone, is not enough to argue in favor of the dilution hypothesis to account for their classification mismatch.

In order to test dilution effects in further details, we computed the $\mathrm{X}$-ray to optical ratio $(2-10 \mathrm{keV}$ band vs. $R$ band) as a function of the unabsorbed, intrinsic $2-10 \mathrm{keV}$ luminosity, as suggested by Fiore et al. (2003) and Eckart et al. (2006). This relation is investigated in Fig. 12, which is restricted to sources with $z<0.8$ because at $z>0.8$, the $R$ band no longer covers the main part of the stellar component longward of the Balmer break (empty+filled squares). However, we also show the unabsorbed type $1 \mathrm{X}$-ray sources lying at $z>0.8$ (stars) as a matter of comparison. We performed a linear fit to those objects that lack broad emission lines in their optical spectra (filled squares) having $L_{2-10}>10^{40} \mathrm{erg} \mathrm{s}^{-1}$. We obtain a correlation with $\left(\chi_{v}^{2}=0.09\right)$ using the $\chi^{2}$ test. We have then compared this value to the $\chi_{v}^{2}$ value obtained if the data are fitted by a constant $\left(\chi_{v}^{2}=0.29\right)$. We then performed an F-test and this test showed that the linear fit is statistically a better description than a 
constant at a highly significant level $\left(P \sim 5.7 \times 10^{-7}\right)$. The bestfit parameters obtained for the linear fit are given by

$\log L_{\mathrm{x}}=0.747( \pm 0.105) \times \log \left(\frac{f_{\mathrm{x}}}{f_{\text {opt }}}\right)-32.48( \pm 4.48)$,

where $L_{\mathrm{x}}$ is the intrinsic X-ray luminosity expressed in erg $\mathrm{s}^{-1}$ and $\frac{f_{\mathrm{x}}}{f_{\mathrm{opt}}}$ is the $\mathrm{X}$-ray to optical flux ratio. This correlation is shown by the solid line in Fig. 12. The objects that lack broad emission lines in their optical spectra (filled squares) thus show a correlation between $\log \left(\frac{f_{\mathrm{x}}}{f_{\text {opt }}}\right)$ and $\log \left(L_{2-10}\right)$, which is expected if the optical light comes predominantly from the host galaxy as opposed to emission from the AGN. Fiore et al. (2003), who found a similar correlation, argue that the observed correlation for the type 2 AGN indicates that the optical light is largely dominated by the host galaxy, due to obscuration. A similar correlation has also been reported by Treister et al. (2005). Indeed, as the X-ray luminosity is mainly coming from the AGN, for low luminosity AGN, the more luminous the AGN is in the $\mathrm{X}$-rays, the larger the ratio $\frac{f_{\mathrm{x}}}{f_{\mathrm{opt}}}$. Note that this observed correlation could be either due to obscuration within the AGN, or to dilution by the host galaxy light. However, as it will be shown in Sect. 5.1.2, dilution effects are more likely than obscuration, as most of the optical spectra are representative of the host galaxy. However, when the AGN is getting much more luminous, its optical light is not diluted anymore by the host galaxy. So for these luminous AGN both the optical and the X-ray components are mainly produced by the AGN. Thus the ratio $\frac{f_{x}}{f_{\text {opt }}}$ is remaining approximately constant as the X-ray luminosity of the AGN is increasing.

This is the reason why the X-ray unabsorbed type 1 do not show the correlation and are clustered at an X-ray luminosity which is significantly higher than the one for the X-ray unabsorbed type 2 AGN. We performed a K-S test in order to test the possible difference in X-ray luminosity for X-ray unabsorbed sources which are either of type 1 or of type 2 . The two sub samples are highly significantly different, the probability that the two are drawn from the same population being almost zero. The type 1 objects have a mean X-ray luminosity $\log \left(L_{2-10}\right)=$ $44.28 \pm 0.55$ and a mean redshift $z=1.38 \pm 0.66$, while the type 2 have a mean X-ray luminosity $\log \left(L_{2-10}\right)=42.68 \pm 0.83$ and a mean redshift $z=0.32 \pm 0.23$. The unabsorbed X-ray sources which are classified as type 2 objects are thus both significantly less luminous than the type 1 objects and are lying at a significantly smaller redshift. Therefore the dilution hypothesis might not be ruled out and is, at least statistically, capable to account for the difference between the X-ray and the optical classifications for these types of X-ray sources. Therefore, the dilution of the AGN light by the host galaxy is mainly occurring at small redshift, supporting the fact that the intrinsic luminosity of the AGN has significantly declined as the redshift is decreasing.

Page et al. (2003) suggested that if the dilution hypothesis holds true, it may explain why a large fraction of unabsorbed $\mathrm{X}$-ray sources lacking broad emission lines in their optical spectra are lying at a low redshift (the majority of our sources of this kind are lying at $z<0.5$ ). The characteristic luminosity of AGN has declined dramatically (by a factor 10) since $z=2$ in both the X-ray (Page et al. 1997) and in the optical (Boyle et al. 2000). So unless the host galaxies of AGN have declined in luminosity by a similar amount, the contrast between the AGN and the host galaxy light will become smaller and smaller as the redshift decreases. The trend that more luminous AGN peak at an earlier era, while the less luminous ones arise later, known as cosmic down-sizing, has also been reported by other authors (see e.g. Eckart et al. 2006; Akylas et al. 2006).

\subsubsection{Line ratio diagrams}

In order to better understand the nature of the unabsorbed X-ray sources, which are only showing narrow emission lines in their optical spectra (type I X-ray sources which are type 2), we have used a refined classification based on the optical spectra. This is based on the diagnostic diagrams of Lamareille et al. (2004), who use blue emission lines ([OII] $\lambda 3727$, [OIII] $\lambda 5007$ and $\mathrm{H} \beta$ ) to discriminate star forming galaxies and HII regions from AGN among intermediate-redshift $(z>$ 0.3 ) objects. This constitutes an improvement compared to the diagram of Veilleux \& Osterbrock (1987) who only used $[\mathrm{OIII}] \lambda 5007 / \mathrm{H} \beta$ vs. [NII] $\lambda 6583 / \mathrm{H} \alpha$, since these two line ratios are only efficient at very low redshifts $(z<0.2)$. Note that very recently, Kewley et al. (2006) have obtained a refined optical classification between pure star forming galaxies, composite galaxies (star forming region+AGN), LINERs and Seyferts. Their refined classification is based on three diagnostic diagrams, namely $[\mathrm{OIII}] \lambda 5007 / \mathrm{H} \beta$ vs. [NII] $\lambda 6583 / \mathrm{H} \alpha$; [OIII] $\lambda 5007 / \mathrm{H} \beta$ vs. [SII] $\lambda 6717 / \mathrm{H} \alpha$ and [OIII] $\lambda 5007 / \mathrm{H} \beta$ vs. [OI] $\lambda 6300 / \mathrm{H} \alpha$. However, these diagrams are only efficient at very low redshifts, as the initial diagram of Veilleux \& Osterbrock (1987). We ran the task splot in the IRAF package onedspec to compute the line ratios. We used the diagrams of both Kewley et al. (2006) and Lamareille et al. (2004), whenever possible. Most of the time the $\mathrm{H} \alpha$ emission line was not present because of the spectral coverage and thus we mainly used the line ratios suggested by Lamareille et al. (2004): [OIII] $\lambda 5007 / \mathrm{H} \beta$ vs. [OII] $\lambda 3727 / \mathrm{H} \beta$.

Figure 13 gathers the line ratios for narrow line AGN presenting both unabsorbed X-ray spectra (filled squares) and absorbed X-ray spectra (empty triangles). More specifically, among the $22 \mathrm{X}$-ray sources upon which we test the dilution hypothesis (22 X-ray sources out of 25 have $L_{2-10}>10^{42} \mathrm{erg} \mathrm{s}^{-1}$ ), $15(68 \%)$ are pure star forming galaxies, $4(18 \%)$ are Seyfert 2, 1 is an absorption line galaxy (ALG, 5\%) and $2(9 \%)$ are undefined (because of the lack of sufficient spectral features to compute line ratios). We have therefore shown that most of the optical spectra for these kinds of sources are more representative of stellar processes in the host galaxy rather than due to the AGN, which constitutes one more argument in favor of the dilution hypothesis. However, we have also found 4 Seyfert 2 which are unabsorbed in the X-rays. Unabsorbed Seyfert 2 have already been found in other samples as the one of Panessa \& Bassani (2002). They claim that these unabsorbed Seyfert 2 might be weak AGN in which the broad line region (BLR) is non-standard (very weak or fading away) or in which the BLR does not exist at all. This scenario is more likely applicable to low luminosity AGN where the brightness of the active nucleus may be insufficient to photoionize the BLR. These 4 Seyfert 2 (i.e. \#9, \#53, \#56 and \#82) have $L_{2-10} \sim 10^{43} \mathrm{erg} \mathrm{s}^{-1}$ and therefore are not considered as low luminosity AGN.

Next, we also computed the line ratios to investigate in further details the nature of the 3 starburst candidates, discussed at the beginning of Sect. 5.1. We have found that source \#13 is a pure star forming galaxy. Its optical spectrum is dominated by the photospheric emission of very hot stars. Thus its X-ray emission could likely be due to the combination of hot gas from supernovae and high mass X-ray binaries in a star-forming galaxy. Source \#55 has been classified as an absorption line galaxy, 

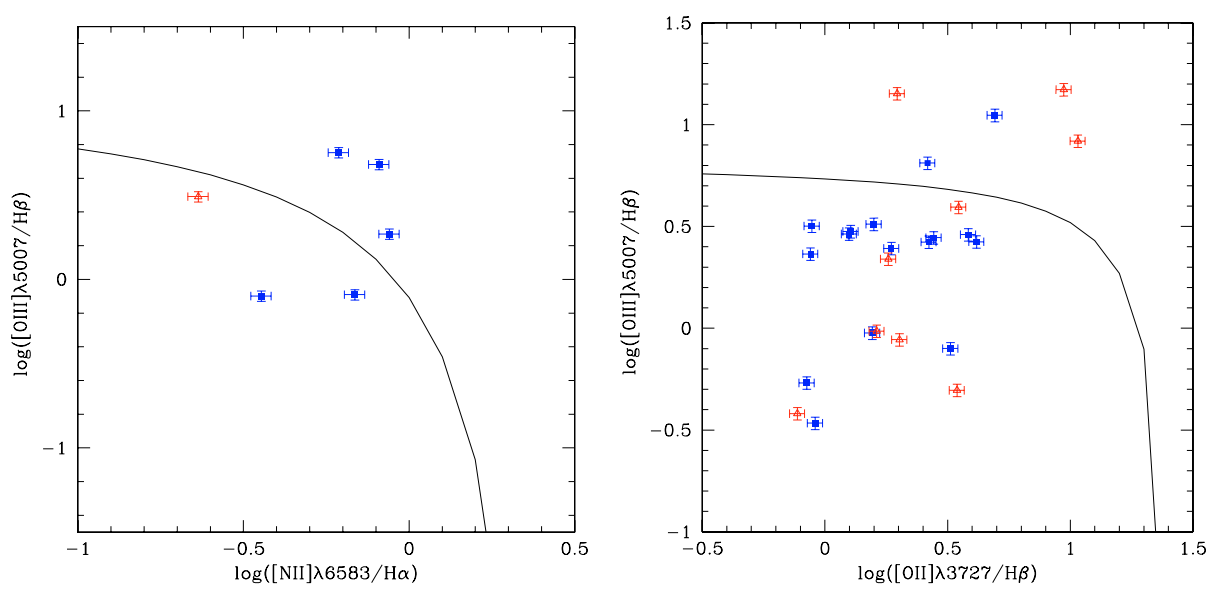

Fig. 13. Diagnostic diagrams based on line ratios for our subsample of narrow line AGN with no absorption in the X-rays (filled squares) and with absorption in the X-rays (empty triangles). 6 of them for which the $\mathrm{H} \alpha$ emission line has been observed, are plotted on the left panel and the other ones are plotted on the right panel. The solid lines from Kewley et al. (2006) (left) and from Lamareille et al. (2004) (right) indicate the separation between star forming galaxies and AGN, the star forming galaxies being below the line and AGN (Seyfert+LINER) above. The error bars have been estimated assuming a $5 \%$ uncertainty for the derived intensities.

which is not consistent with the starburst hypothesis. And thus, this X-ray source is more likely a LLAGN which is heavily diluted by the light of its host galaxy. Finally, source \#63 has been classified as a Seyfert 2 from its optical line ratios, instead of a starburst. This X-ray source is thus a low luminosity AGN similar to those detected by Panessa \& Bassani (2002), in which the BLR might be very weak or fading away. On the other hand, the source \#63 could also be a good Compton thick candidate, as a Fe emission line has been detected in its X-ray spectrum. In this case, we only observe the indirect reflected component of the X-ray emission, and thus both its intrinsic X-ray luminosity and hydrogen column density would have been highly underestimated. One way to test the Compton thick nature of this AGN would be to plot the equivalent width of the Fe line against the $\frac{F_{\mathrm{x}}}{F_{[\mathrm{OIII}]}}$ ratio (see Fig. 1 of Bassani et al. 1999). Unfortunately, we have not been able to compute $F_{[\mathrm{OIII}]}$, as our optical spectra are only calibrated in relative fluxes. Thus, out of the 3 starburst candidates, only 1 is a starburst galaxy. Of course, the above 4 unabsorbed Seyfert 2 could also be Compton thick candidates. However, as no Fe line have been detected in their X-ray spectra, this hypothesis is much less probable to take into account their nature.

Finally, we have computed the line ratios for the absorbed $\mathrm{X}$-ray sources which are only presenting narrow emission lines in their optical spectra (type II X-ray sources which are type 2). As can be seen in Table 2, there are 13 such sources. 5 of them (38\%) are Seyfert 2. Figure 13 shows only $3 / 5$ in the AGN region of the diagrams. However, there are also two (empty triangles) slightly below the dividing line (thus in the star forming galaxy region) that have been classified as Seyfert 2 anyway, because of the presence in their optical spectra of the emission line of $[\mathrm{NeIII}] \lambda 3869$, which is a line typical of AGN. 1 is an absorption line galaxy (8\%), 5 are pure star forming galaxies $(38 \%)$, and 2 are unidentified (16\%). Note that 1 of the 2 unidentified objects is a NELG, and coupled with its X-ray characteristics has been classified as a type II QSO. So, for a majority of these $13 \mathrm{X}$-ray sources (7/13), the optical spectra are AGN-like, rather than resembling the one of the host galaxy. Therefore the absorbed X-ray sources which are only presenting narrow emission lines are truly hidden AGN.

Table 3 shows an overview of the refined classification of the obscured X-ray sources, which is based on the emission line ratios.

Finally, Fig. 14 shows two examples of absorbed X-ray sources which only present narrow emission lines in their optical spectra.
Table 3. Refined classification of the type 2 sources (both type I and type II), which is based on the emission line ratios computed from their optical spectra.

\begin{tabular}{cccc}
\hline \hline & Type I 2 & Type II 2 & $N$ \\
\hline SF glx & 16 & 5 & 21 \\
Sy 2 & 5 & 5 & 10 \\
ALG & 2 & 1 & 3 \\
Undef & 2 & 2 & 4 \\
\hline $\mathrm{N}$ & 25 & 13 & $\mathbf{3 8}$ \\
\hline
\end{tabular}

\subsubsection{Testing the dilution hypothesis}

Until now we have presented several observational arguments in favor of the dilution of the AGN optical light by the host galaxy light. We now develop a simple model in order to investigate whether dilution effects are plausible or not. In this model, we consider the contributing optical flux in a 1 " slit from the host galaxy and, first, from X-ray unabsorbed AGN only presenting narrow emission lines $\left(L_{2-10} \sim 5 \times 10^{42} \mathrm{erg} \mathrm{s}^{-1} ; z \sim 0.3\right)$ and from X-ray unabsorbed AGN presenting broad emission lines $\left(L_{2-10} \sim 2 \times 10^{44} \mathrm{erg} \mathrm{s}^{-1} ; z \sim 1.5\right)$. In order to convert X-ray flux into optical flux, we take a typical ratio $\frac{f_{\mathrm{x}}}{f_{\mathrm{opt}}}=1$. Next, we model the host galaxy with $M_{R}=-22 .(R \sim 19$ for $z \sim 0.3)$ and with a physical size of $30 \mathrm{kpc}$. Finally, we apply the corresponding k-correction for a typical elliptical galaxy (Coleman et al. 1980), and we use the galaxy profile of Burkert et al. (1993) in order to estimate the fraction of the total optical flux of the host galaxy which enters the slit. Making the reasonable assumption that the typical absolute magnitude of the host galaxy of radio quiet AGN is similar at $z=0.3$ and at $z=1.5$, (Kukula et al. 2001, Fig. 7), we derive the following values:

$$
\begin{aligned}
& \text { - At } z=0.3, \\
& \quad\left(\frac{F_{\text {host,slit }}}{F_{\mathrm{AGN}}}\right)_{R} \sim 2, \\
& \text { - At } z=1.5, \\
& \quad\left(\frac{F_{\text {host }, \text { slit }}}{F_{\mathrm{AGN}}}\right)_{R} \sim \frac{1}{400},
\end{aligned}
$$

where $F_{\text {host,slit }}$ is the fraction of the host galaxy light entering the slit and $F_{\mathrm{AGN}}$ is the AGN light entering the slit. Note that other previous works have claimed that the host galaxies of AGN were about 2.5 mag brighter at $z \simeq 2$ than those of low-redshift AGN (see e.g. Aretxaga et al. 1998). But even taking this into account, 

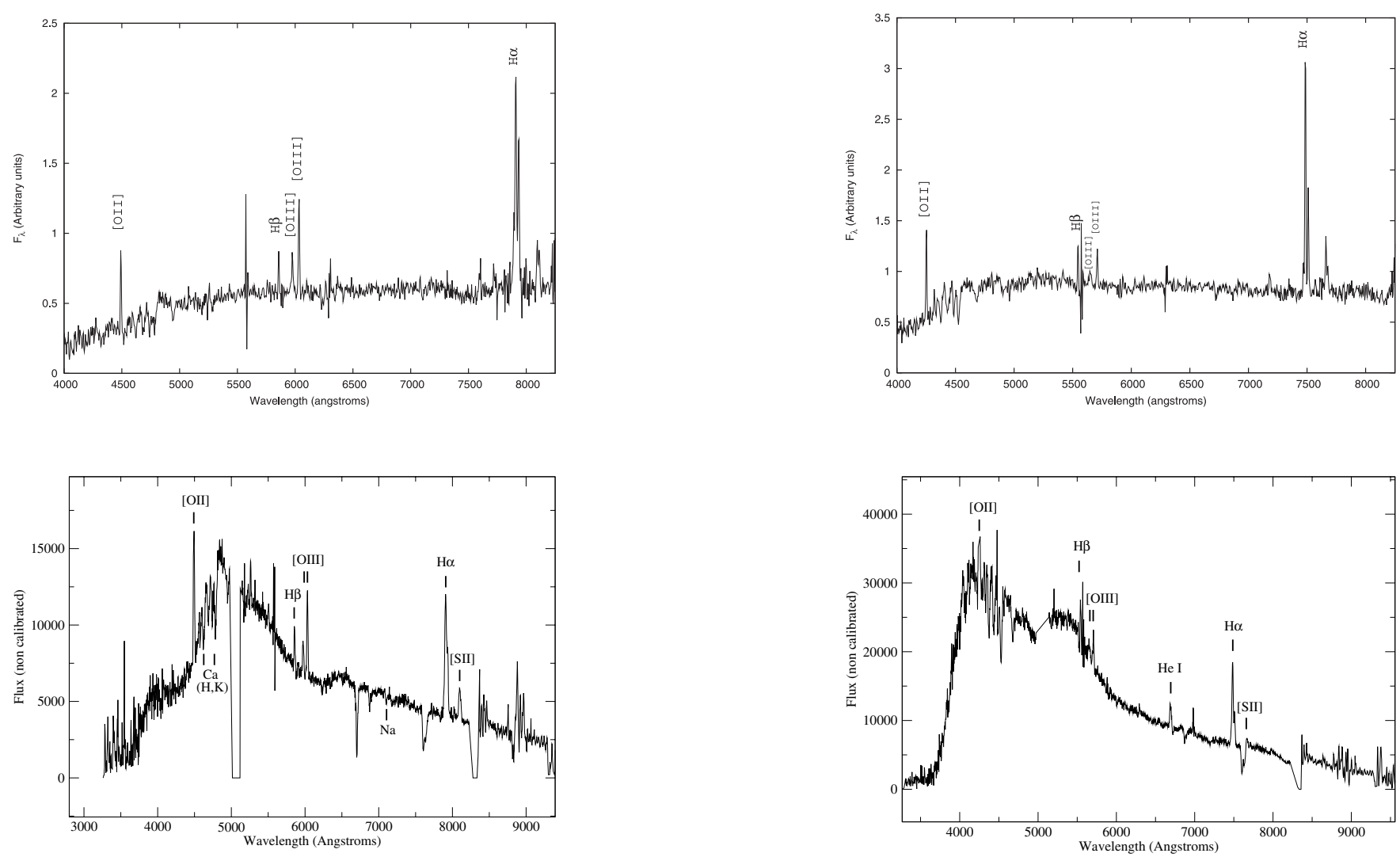

Fig. 14. $2 \mathrm{dF}$ spectra of two absorbed X-ray sources which only present narrow emission lines in their optical spectra (upper panel). These are sources \#84 and \#66 in Table 4, respectively. They have been classified as star forming galaxies, based on the line ratios inferred from their optical spectra. The lower panel show the optical spectra of the corresponding objects, obtained with the SALT. Both have been observed with the PG0300 grism $(R \sim 400)$. The SALT optical spectra are not flux calibrated.

the AGN at $z \simeq 1.5$ would still be about 40 times brighter than their host galaxies, thus preventing dilution effects.

Thus for the above AGN around $z=0.3$, the flux of the host galaxy entering into the slit is about twice as large as the optical flux of the AGN. On the other hand, for high redshift AGN $(z=1.5)$, the optical flux of the AGN is totally overwhelming the optical flux of the host galaxy and dilution effects can not occur anymore. Even if crude, this model allows us to confirm that dilution can definitely be present in low luminosity and low redshift AGN, if host galaxy properties do not evolve significantly with the redshift.

As a conclusion, in this section, we have shown that dilution effects can very possibly explain the difference between the $\mathrm{X}$-ray and the optical classifications of a great majority of these $25 \mathrm{X}$-ray sources. Among these $25 \mathrm{X}$-ray sources, we have also found 5 Seyfert 2 ( 4 with $L_{2-10}>10^{42} \mathrm{erg} \mathrm{s}^{-1}$, and 1 with $L_{2-10}<$ $10^{42}$ erg s$^{-1}$ ) which do not present strong X-ray absorption in their X-ray spectra.

Therefore, apart from these $5 \mathrm{X}$-ray unabsorbed Seyfert 2, the dilution hypothesis works, without any need to alter the standard orientation-based AGN unified scheme.

\subsection{Absorbed AGN showing broad emission lines in their optical spectra}

Figure 15 illustrates a representative example of an absorbed $\mathrm{X}$-ray source with bright and obvious broad emission lines in its optical spectrum and so with no hint of obscuration in the optical. This is source \#20 in Table 4. We have found 7 such X-ray sources.
These $7 \mathrm{X}$-ray sources are only found in the high luminosity regime of $\mathrm{AGN}\left(L_{2-10}>6 \times 10^{43} \mathrm{erg} \mathrm{s}^{-1}\right), 4$ of them having an $L_{X}$ typical of QSOs. Because of their high X-ray luminosity, dilution effects are totally ruled out to account for their nature. Therefore these 7 objects are not consistent with the standard orientation-based unified scheme. They span a broad range in redshift $(z=[0.4-3.6])$, most of them being high redshift AGN.

In Fig. 11, these 7 sources (filled triangles) have X-ray/optical ratios typical of AGN which are both unabsorbed in the $\mathrm{X}$-rays and which present broad emission lines in their optical spectra (empty squares). In order to investigate this in further details, we have compared the average X-ray luminosity of these absorbed AGN being type 1 with the one of the unabsorbed AGN, also being type 1 . The average X-ray luminosities are $\log L_{2-10}=44.59 \pm 0.56$ and $\log L_{2-10}=44.28 \pm 0.55$, respectively, which turns out to be quite similar. We also compared the average spectroscopic redshifts for these 2 classes. We obtain $z=1.93$ and $z=1.38$, respectively, both representative of high redshift AGN. The above 2 classes of objects have on average the same intrinsic X-ray luminosity and redshift, and are both lying in the high luminosity/high redshift regime. These two types of objects thus seem to belong to the same AGN population.

Such anomalous objects have already been observed by several authors (e.g. Perola et al. 2004; Eckart et al. 2006). They respectively find that around $5 \%$ and $15 \%$ of the X-ray sources in their respective sample are X-ray absorbed sources which present broad emission lines in their optical spectra. Both these values bracket the fraction that we have found, which is around $7 \%$. Interestingly, these two studies also find that these X-ray absorbed sources with broad emission lines in their optical spectra 

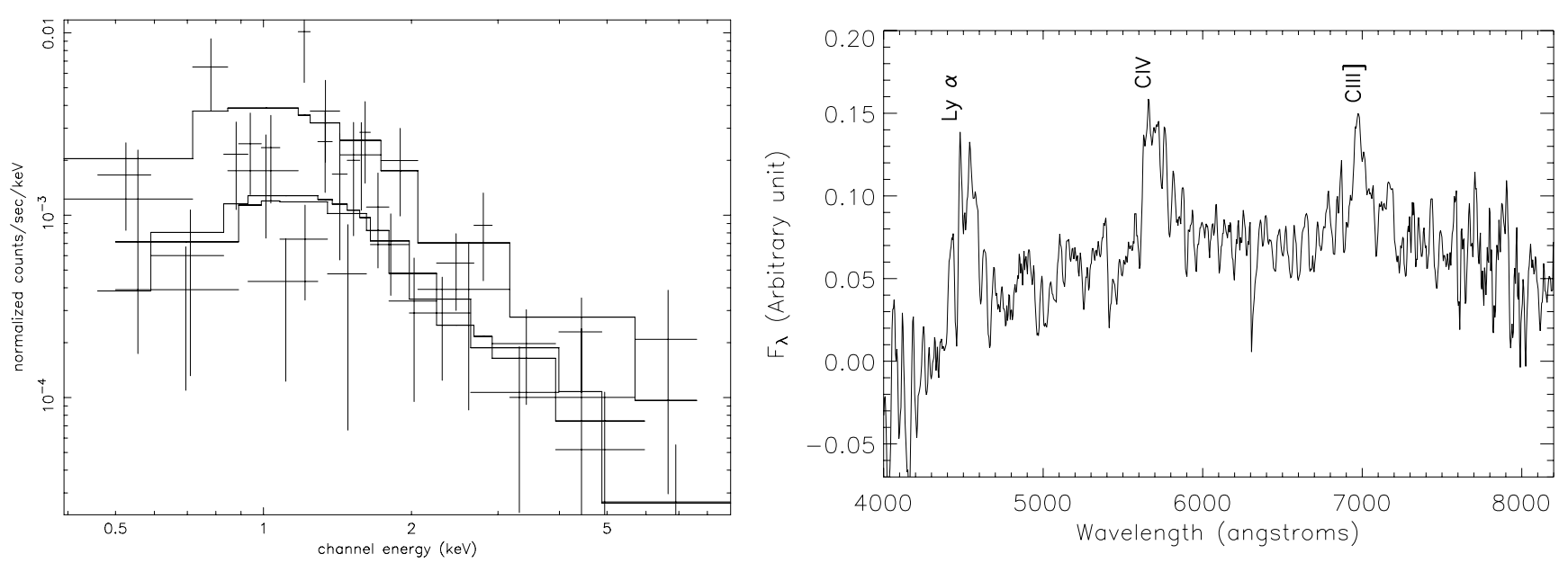

Fig. 15. MOS1 MOS2 (the two lower functions) and pn (upper one) X-ray spectra of a source which is showing strong X-ray absorption with $N_{\mathrm{H}}^{\mathrm{int}}=4 \times 10^{22} \mathrm{~cm}^{-2}$. The crosses represent the data points and the solid line represent the folded model simultaneously fitted to the data of the 3 detectors (left). The optical counterpart has been identified as a BLAGN lying at a redshift $z=2.66$ (right). This is source \#20 in Table 4.

are high luminosity AGN lying at high redshift (typically $z>1$ ). So it seems that high luminosity and high redshift are common properties of these peculiar objects.

Several interpretations have been suggested in order to explain their nature. Maiolino et al. (2001a) have presented a sample of 19 nearby AGN whose X-ray spectra show evidence for cold absorption and no hint of obscuration in the optical (hence classified as type 1 ). They concluded that the $\frac{E_{B-V}}{N_{\mathrm{H}}}$ ratio is systematically much lower than the Galactic standard value. In a companion paper, Maiolino et al. (2001b) suggest that a dust distribution dominated by large grains in the obscuring torus could explain the low $\frac{E_{B-V}}{N_{\mathrm{H}}}$ values obtained. They claim that the formation of large grains is naturally expected in the high density environment characterizing the circumnuclear region of AGN. These large grains make the extinction curve flatter than the galactic one and thus for a given $N_{\mathrm{H}}$ a reduced extinction and reddening are observed, compared to the galactic standard.

Alternatively, Weingartner \& Murray (2002) propose a model to explain these absorbed AGN presenting broad and obvious emission lines in their optical spectra. They show that the large grain hypothesis in the obscuring torus is not needed in order to explain most of the sample of Maiolino et al. (2001a): they suggest that the material that absorbs the X-rays is probably unrelated to the material that absorbs the optical/infrared radiation and that the torus is probably not probed by the observations of Maiolino et al. (2001a). They suggest that the line of sight of the sample of Maiolino et al. (2001a) passes through ionized material located just off the torus and/or accretion disk. This material is responsible for the X-ray absorption, while the optical/infrared extinction occurs in material farther from the nucleus, where the dust may be quite similar to galactic dust. The $\mathrm{X}$-ray-absorbing material may be dust-free or may contain large grains that have very small extinction efficiencies in the opti$\mathrm{cal} /$ infrared. This material may be associated with a disk wind, which would originate within the dust sublimation radius (see Murray et al. 1995). In this case, the dust will sublimate and the obscuration/extinction in the optical will be much reduced, even if there is a strong absorption in the X-rays, produced by the ionized gas. Note that according to this model, the optical spectra of this type of sources should also present broad absorption lines, and thus be classified as BAL QSOs, because of the wind outflow.
Recent works suggest that BAL QSOs (which are high luminosity AGN with broad absorption and emission lines in their optical spectra) are weak soft X-ray sources, the X-ray weakness being attributed to absorption rather than being intrinsic (Brandt et al. 2000; Punsly 2006). Thus most of the BAL QSOs can also be classified as X-ray absorbed sources which present broad emission lines in their optical spectra. Therefore, it is tempting to suggest that these QSOs (which are optically selected) are actually the same population of objects than the X-ray absorbed type 1 AGN, detected in X-ray surveys.

However, generally, only up to a few percent of the X-ray absorbed sources with broad emission lines are identified as a BAL QSO: none of our 7 X-ray sources have an optical spectrum consistent with a BAL QSO spectrum. Similarly, Perola et al. (2004) do not report any BAL QSOs among the X-ray absorbed type 1 AGN from their sample. Finally, Eckart et al. (2006) find that only $14 \%$ of these sources are BAL QSOs. Thus generally, these two types of AGN do not seem to belong to the same AGN populations. Indeed, Risaliti et al. (2001) have shown that only a fraction of the absorbed X-ray sources with broad emission lines, detected in X-ray surveys, could also actually be classified as BAL QSOs, with the latter constituting the tail of the population of X-ray weak quasars. However, note that, as BAL occur mainly in the UV, blueward of the CIV emission line, the spectral coverage of the optical spectra does only allow the observation of the broad CIV absorption line for high $z$ AGN (typically $z>1.6$ for the blue part of the spectrum starting at $4000 \AA$ ). Moreover, the broad absorption lines could be difficult to detect due to the moderate level of extinction and generally require high signal-to-noise ratio optical spectra. Thus the fact that X-ray absorbed type 1 AGN and BAL QSOs have common properties is not totally ruled out.

One way to choose between the large grain hypothesis and the low dust-to-gas ratio would be to fit the X-ray spectra of the 7 objects with models including gas being highly ionized and check if they fit better the X-ray spectra than a model with neutral gas. Unfortunately, we have not been able to test it, as our $\mathrm{X}$-ray spectra do not have a signal to noise ratio high enough.

Alternatively, these $7 \mathrm{X}$-ray sources could be at a different evolutionary stage: the fact that the absorbed AGN presenting broad emission lines in their optical spectra are only found in the high luminosity/redshift regime of the AGN is consistent with the scenario suggested by Page et al. (2004). They have observed 
8 absorbed AGN presenting broad emission lines in their optical spectra in the submillimeter domain with SCUBA and showed that these QSOs are characterized by a much stronger submillimeter luminosity compared to a sample of 20 unabsorbed AGN presenting broad emission lines in their optical spectra. They claim that this can be understood as an isotropic signature of copious star formation, with the QSO being embedded in the dense interstellar media of their forming host spheroids. This rules out orientation effects as the cause of the absorption. Very recently, Page et al. (2006b) have suggested that the X-ray absorption of these objects is most likely due to a dense ionized wind driven by the QSO. This wind could be the mechanism by which the QSO terminates the star formation in the host galaxy, and ends the supply of accretion material, to produce the present day black hole/spheroid mass ratio.

Therefore they suggest that the absorbed and unabsorbed QSOs represent different stages in an evolutionary sequence, in which the absorbed QSOs represent the earlier phase. They estimate that the absorbed phase typically lasts only around $15 \%$ of the unabsorbed phase. In our sample, we have found 61 type 1 AGN. 7 of them are presenting strong X-ray absorption. So these objects represent about $11 \%$ of the type 1 AGN. This is consistent (within $1 \sigma$ ) with the value predicted by Page et al. (2004). However, we would need to detect many more objects of this kind in order to better understand their nature and to test in further details the above model.

We finally compared the X-ray luminosity and redshift for the X-ray absorbed AGN classified as type 1 (with broad emission lines in their optical spectra) and type 2 (only with narrow emission lines in their optical spectra). The mean X-ray luminosities are $\log L_{2-10}=44.59 \pm 0.56$ and $\log L_{2-10}=42.89 \pm$ 0.69 , respectively. Concerning the redshifts, the corresponding mean values are $z=1.93$ and $z=0.317$, respectively. So, when comparing these two classes, we notice that they differ significantly, both in redshift and luminosity: the absorbed AGN presenting broad emission lines in their optical spectra are both more luminous in the X-rays and lying at a significantly higher redshift than the ones only presenting narrow emission lines in their optical spectra.

As a conclusion, we have confirmed the existence of a population of AGN which are classified as type II in the X-rays and as type 1 in the optical. This population could actually be X-ray unabsorbed type $1 \mathrm{X}$-ray sources, intrinsically (either they are at a different evolutionary stage, or their dust is sublimated; either way, we can not rule out the presence of an intrinsic type 1 QSO, surrounded by a torus).

\section{X-ray bright optically normal galaxies (XBONG)}

The existence of an intriguing population of galaxies with X-ray properties suggesting the presence of an AGN, but without any obvious sign of activity in their optical spectra, has been known for more than 20 years (e.g. Elvis et al. 1981; Griffiths et al. 1995; Severgnini et al. 2003). The nature of this kind of sources is far from being understood. Different scenarios have been proposed so far: a) the AGN is a BL Lac object; b) the nucleus activity is outshone by the stellar continuum of the host galaxy (as shown by Severgnini et al. 2003); c) the emission lines could be absorbed by material beyond the NLR. Most of these optically dull galaxies seem to be absorbed in the X-rays (Mainieri et al. 2002). Comastri et al. (2003) have recently shown that the distribution of X-ray-to-optical flux ratio of XBONG sources could be well reproduced assuming that the underlying SED of the putative AGN is that of an X-ray Compton-thick AGN.

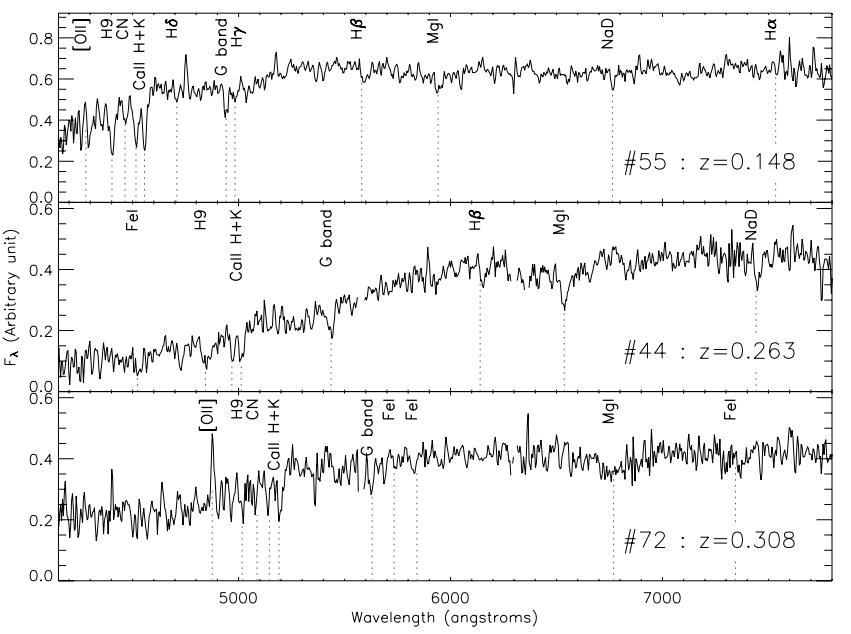

Fig. 16. The $3 \mathrm{XBONG}$ candidates. Each of these 3 XBONG lacks emission lines, except for [OII] in source \#72. This source presents strong absorption in the X-rays $\left(N_{\mathrm{H}}^{\mathrm{int}}=3.86 \times 10^{22} \mathrm{~cm}^{-2}\right)$.

In our sample of X-ray sources, 3/99 have been classified as absorption line galaxies (see Fig. 16). The X-ray properties of these $3 \mathrm{X}$-ray sources suggest the presence of AGN activity: they have an intrinsic luminosity $L_{2-10}>9 \times 10^{41} \mathrm{erg} \mathrm{s}^{-1}$, two of them having $\frac{F_{\mathrm{x}}}{F_{\mathrm{opt}}}>0.1$, similar to typical values of luminous AGN (Fiore et al. 2003). So these 3 X-ray sources are good XBONG candidates. Two of them (source \#44 and source \#55) are unabsorbed in the X-rays. Dilution effects could be responsible for the lack of emission lines in the optical spectra of these two X-ray sources, as they lie on the relation given by Eq. (4). Finally, source \#72 presents strong absorption in the X-rays $\left(N_{\mathrm{H}}^{\text {int }}>10^{22} \mathrm{~cm}^{-2}\right.$ at the $95 \%$ confidence level). For this source, strong absorption along with some dilution effects could explain why this source lacks AGN features in its optical spectrum and is thus classified as XBONG.

These 3 X-ray sources have similar properties to some XBONG that are emerging from different X-ray surveys (e.g. Severgnini et al. 2003), although a closer look at their spectra, based on high resolution data and/or on a better spectral coverage might reveal their real AGN nature. The finding of these 3 XBONG candidates clearly shows that optical spectroscopy sometimes can be inefficient in revealing the presence of an AGN, which instead becomes obvious from an X-ray spectroscopic investigation.

\section{Type II QSO candidates}

In this work, we have discovered $6 \mathrm{X}$-ray sources with $N_{\mathrm{H}}^{\mathrm{int}}>$ $10^{22} \mathrm{~cm}^{-2}$ and $L_{2-10}>10^{44} \mathrm{erg} \mathrm{s}^{-1}$. However, 5 of these sources have type 1 optical spectra (e.g. with broad emission lines). The remaining object (source \#42), classified as a type 2 QSO from its optical spectrum, is therefore the only genuine type II QSO detected amongst our sample of $99 \mathrm{X}$-ray sources. This object is lying at a rather high redshift $(z \sim 1)$.

Recently, a connection between Extremely Red Objects (EROs) and type II QSOs has been suggested (see e.g. Gandhi et al. 2004; Severgnini et al. 2005). The UKIDSS $K$ band magnitude of our type 2 QSO candidate is $K \sim 17.2$ and its color $R$ $-K$ is equal to 4.6. It is thus very close to the $R-K>5$ threshold adopted to define the population of EROs. Note that this type II QSO candidate has independently been reported by 
Tajer et al. (2007) in fitting photometric data points with several SED templates. We confirm here the nature of this source.

\section{Summary and conclusions}

We have presented and characterized a sample of $99 \mathrm{X}$-ray point sources for which we have secure identification and spectroscopic redshift. These $99 \mathrm{X}$-ray sources have been selected in the $[2-10] \mathrm{keV}$ band with at least 80 counts in the $[0.5-10] \mathrm{keV}$ band, from a sample of $612 \mathrm{X}$-ray sources.

A large fraction of our X-ray sources (about 95\%) have been identified as AGN. We have analyzed their optical classification by measuring the $F W H M$ of the emission lines present in their optical spectra. In particular we have divided the X-ray sources in 61 type 1 AGN (BLAGN) and 38 type 2 AGN (35 NELGs+3 ALGs). Next we have fitted the X-ray spectra of these sources and inferred their hydrogen column density. Proceeding this way, we have found 79 type I X-ray sources $\left(N_{\mathrm{H}}^{\text {int }}<10^{22} \mathrm{~cm}^{-2}\right)$ and 20 type II X-ray sources $\left(N_{\mathrm{H}}^{\text {int }} \geq 10^{22} \mathrm{~cm}^{-2}\right)$. The X-ray and the optical properties have been analyzed and intercompared. The main results of our work can be summarized as follows:

- We have shown that there is at most a mild correlation between the X-ray and the optical classifications, 32 X-ray sources out of 99 having different X-ray and optical properties. Then we have shown that this discrepancy is not a function of the X-ray flux and luminosity. These results agree with several other published studies.

- We have gathered several pieces of evidence in favor of dilution effects, where the AGN light is overshone by a luminous host galaxy, for a vast majority of the 25 unabsorbed X-ray sources which only present narrow emission lines in their optical spectra. This is a nice confirmation of previously published results (e.g. Severgnini et al. 2003; Silverman et al. 2005). We have also found 5 Seyfert 2 which do not present a strong absorption in the X-rays. Only 1 of them is a good Compton thick AGN candidate.

- We have reported 7 absorbed X-ray sources which present broad emission lines in their optical spectra. This corresponds to $7 \%$ of the whole sample and to $11 \%$ of the type 1 AGN. These objects, which are highly luminous AGN (mostly QSOs) and lie at large redshifts, have similar X-ray luminosity and redshift as the unabsorbed X-ray sources which present broad emission lines in their optical spectra. The difference between the X-ray and the optical classifications of these $7 \mathrm{X}$-ray sources could easily be accounted for if the gas responsible for the X-ray absorption is highly ionized, instead of being neutral, in which case the accompanying dust would sublimate to yield a much smaller dust-to-gas ratio, resulting in a reduced optical extinction and reddening. However, our X-ray spectra do not have a signal to noise ratio high enough to distinguish ionized gas model from neutral gas models. Moreover, part of these sources could be BAL QSOs. From this perspective, it seems plausible that the obscuring material in these sources has different composition than typical Seyferts (e.g. either a very low dust: gas ratio, or a dust-free wind).

- Our study, which has been done in parallel to the work of Tajer et al. (2007), has allowed us to confirm their main results, but using a safer procedure as we utilized spectroscopic redshifts along with secure spectroscopic identifications, instead of photometric redshifts obtained by fitting photometric data points with SED templates. Moreover, our study covers a much larger area.
- We have found 1 genuine type II QSO candidate amongst our X-ray sample. Note that we have also identified $5 \mathrm{X}$-ray absorbed QSOs which are not obscured in the optical.

As a conclusion, most of the discrepancy between the X-ray and the optical classifications comes from the fact that type 2 optical sources are more likely to be unabsorbed than absorbed in the X-rays. We have shown that the vast majority of these type 2 optical sources which are unabsorbed in the X-rays are affected by dilution effects, which do not require any modification of the standard orientation-based AGN unification scheme. Thus this AGN unification scheme still holds for about $88 \%$ of the X-ray sources in our sample, as their predictions are not met for only $12 / 99$ of the X-ray sources. Note that the discrepancy between the absorption properties in the X-rays and in the optical/UV that we have found, could as well be a natural consequence of the clumpy nature of the absorbing medium, as suggested by Elitzur (2006): the torus could be made of individual clouds, with the "X-ray torus" being different from the "dusty torus", instead of a continuous absorbing medium with sharp edges.

As shown at the beginning of the paper, we are biased against faint optical counterparts $(R \geq 22)$. It would thus be very interesting to extend our work to significantly fainter optical counterparts in order to check whether the fraction of X-ray sources for which the X-ray and the optical classifications do not match is still that large.

Acknowledgements. We thank the referee for useful suggestions. We are indebted to D. Hutsemékers for some fruitful discussions. This work is based on observations obtained with XMM-Newton, an ESA science mission with instruments and contributions directly funded by ESA Member States and NASA. OG, EG, PGS, VB, and JS acknowledge support from the ESA PRODEX Programme "XMM-LSS", and from the Belgian Federal Science Policy Office for their support. LC, DM, MT acknowledge financial contribution from contract ASI-INAF I/023/05/0. PG is a Fellow of the Japan Society for the Promotion of Science. MK acknowledges the German DLR under grant 50OR0404. ACR, JEC and FJC acknowledge financial support from the Spanish Ministry for Education and Science, under projects ESP2003-00812 and ESP2006-13608-C02-01. JAT, SM, SR and MW acknowledge support from the UK PPARC research council.

\section{References}

Akylas, A., Georgantopoulos, I., Georgakakis, A., et al. 2006, A\&A, 459, 693 Alexander, D. M., Bauer, F. E., Brandt, W. N., et al. 2003, AJ, 126, 539 Antonucci, R. 1993, ARA\&A, 31, 473

Aretxaga, I., Terlevich, R. J., \& Boyle, B. J. 1998, MNRAS, 296, 643 Barcons, X., Carrera, F. J., \& Ceballos, M. T. 2003, MNRAS, 339, 757 Bassani, L., Dadina, M., Maiolino, R., et al. 1999, ApJ, 121, 473 Bondi, M., Ciliegi, P., Zamorani, G., et al. 2003, A\&A, 403, 857 Boyle, B. J., Shanks, T., Croom, S. M., et al. 2000, MNRAS, 317, 1014 Brandt, W. N., Laor, A., Wills, B. J., et al. 2000, ApJ, 528, 637 Burkert, A. 1993, A\&A, 278, 23

Caccianiga, A., Severgnini, P., Braito, V., et al. 2004, A\&A, 416, 901

Carrera, F., Ebrero, J., Mateos, S., et al. 2007, A\&A, 469, 27

Cash, W. 1979, ApJ, 228, 939

Chiappetti, L., Tajer, M., Trinchieri, G., et al. 2005, A\&A, 439, 413

Cohen, A. S., Röttgering, H. J. A., \& Kassim, N. E. 2003, ApJ, 591, 640

Coleman, G. D., Wu, C. C., \& Weedman, D. W. 1980, ApJS, 43, 393

Comastri, A., Setti, G., Zamorani, G., et al. 1995, A\&A, 296, 1

Comastri, A., Fiore, F., Vignali, C., et al. 2001, MNRAS, 327, 781

Comastri, A., Brusa, M., Mignoli, M., \& the HELLAS2XMM colaboration 2003, AN, 324, 28

Della Ceca, R., Maccacaro, T., Caccianiga, A., et al. 2004, A\&A, 428, 383

Dickey, J. M., \& Lockman, F. J. 1990, ARA\&A, 28, 215

Downes, A. J. B., Peacock, J. A., Savage, A., et al. 1986, MNRAS, 218, 31

Dye, S., Warren, S. J., Hambly, N. C., et al. 2006, MNRAS, 372, 1227

Eckart, M. E., Stern, D., Helfand, D. J., et al. 2006, ApJS, 165, 19

Elitzur, M. 2006, ApJS, The Central Engine of Active Galactic Nuclei, ed. L. C. Ho, \& J.-M. Wang [arXiv: astro-ph/0612458], in press

Elvis, M., Schreier, E. J., Tonry, J., et al. 1981, ApJS, 246, 20

Fiore, F., La Franca, F., Giommi, P., et al. 1999, MNRAS, 306, L55 
Fiore, F., Brusa, M., Cocchia, F, et al. 2003, A\&A, 409, 79

Gallagher, S. C., Brandt, W. N., Chartas, G., et al. 2006, ApJ, 644, 709 Gandhi, P., \& Fabian, A. C. 2003, MNRAS, 339, 1095

Gandhi, P., Crawford, C. S., Fabian, A. C., et al. 2004, MNRAS, 348, 529

Gandhi, P., Garcet, O., Disseau, L., et al. 2006, A\&A, 457, 393

Gavignaud, I., Bongiorno, A., Paltani, S., et al. 2006, A\&A, 457, 79

Gehrels, N. 1986, ApJ, 303, 336

Giacconi, R., Zirm, A., Wang, J., et al. 2002, ApJS, 139, 369

Gilli, R., Comastri, A., \& Hasinger, G. 2007, A\&A, 463, 79

Griffiths, R. E., Georgantopoulos, I., Boyle, B. J., et al. 1995, MNRAS, 275, 77

Hickox, R. C., \& Markevitch, M. 2006, ApJ, 645, 95

Kewley, L. J., Groves, B., Kauffmann, G., et al. 2006, MNRAS, 372, 961

Kukula, M. J., Dunlop, J. S., McLure, R. J., et al. 2001, MNRAS, 326, 1533

Lamareille, F., Mouhcine, M., Contini, T., et al. 2004, MNRAS, 350, 396

Lawrence, A., Warren, S. J., Almaini, O., et al. 2007, MNRAS, 379, 1599

Le Fèvre, O., Mellier, Y., McCracken, H. J., et al. 2004, A\&A, 417, 839

Le Fèvre, O., Vettolani, G., Garilli, B., et al. 2005, A\&A, 439, 845

Lonsdale, C. J., Smith, H. E., Rowan-Robinson, M., et al. 2003, PASP, 115, 897

Mainieri, V., Bergeron, J., Hasinger, G., et al. 2002, A\&A, 393, 425

Mainieri, V., Hasinger, G., Cappelluti, N., et al. 2006, ApJS COSMOS [arXiv: astro-ph/0612361], accepted

Maiolino, R., Marconi, A., Salvati, M., et al. 2001a, A\&A, 365, 28

Maiolino, R., Marconi, A., \& Oliva, E. 2001b, A\&A, 365, 37

Mateos, S., Barcons, X., Carrera, F. J., et al. 2005, A\&A, 433, 855

Moran, E. C., Filippenko, A. V., \& Chornock, R. 2002, ApJ, 579, L71

Murray, N., Chiang, J., Grossman, S. A., et al. 1995, ApJ, 451, 498

Nandra, K., \& Pounds, K. A. 1994, MNRAS, 268, 405

Pacaud, F., Pierre, M., Refregier, A., et al. 2006, MNRAS, 372, 578

Page, M. J., Mason, K. O., McHardy, I. M., et al. 1997, MNRAS, 291, 324

Page, M. J., Mittaz, J. P. D., \& Carrera, F. J. 2001, MNRAS, 325, 575
Page, M. J., McHardy, I. M., \& Gunn, K. F. 2003, AN, 324, 101

Page, M. J., Stevens, J. A., Ivison, R. J., et al. 2004, ApJ, 611, L85

Page, M. J., Loaring, N. S., Dwelly, T., et al. 2006a, MNRAS, 369, 156

Page, M. J., Carrera, F. J., Ebrero, J., et al. 2006b, in conference proceedings, Studying Galaxy Evolution with Spitzer and Herschel, in press

Panessa, F., \& Bassani, L. 2002, A\&A, 394, 435

Pappa, A., Georgantopoulos, I., Stewart, G. C., et al. 2001, MNRAS, 326, 995

Perola, G. C., Puccetti, S., Fiore, F., et al. 2004, A\&A, 421, 491

Pierre, M., Valtchanov, I., Santos, S. D., et al. 2004, JCAP, 9, 11

Pierre, M., Pacaud, F., Duc, P. A., et al. 2006, MNRAS, 372, 591

Pierre, M., Chiappetti, L., Pacaud, F., et al. 2007, MNRAS

[arXiv:astro-ph/07083299v1]

Polletta, M., Tajer, M., Maraschi, L., et al. 2007, ApJ, 663, 81

Punsly, B. 2006, ApJ, 647, 886

Ranalli, P., Comastri, A., \& Setti, G. 2003, A\&A, 399, 39

Risaliti, G., Marconi, A., Maiolino, G., et al. 2001, A\&A, 371, 37

Setti, G., \& Woltjer, L. 1989, A\&A, 224, L21

Severgnini, P., Caccianiga, A., \& Braito, V. 2003, A\&A, 406, 483

Severgnini, P., Della Ceca, R., \& Braito, V. 2005, A\&A, 431, 87

Silverman, J. D., Green, P. J., Barkhouse, W. A., et al. 2005, ApJ, 618, 123

Spergel, D. N., Bean, R., Dore, O., et al. 2007, ApJ, 170, 377

Tajer, M., Polletta, M., Chiappetti, L., et al. 2007, A\&A, 467, 73

Treister, E., \& Urry, M. 2005, ApJ, 630, 115

Treister, E., Castander, F. J., Maccarone, T. J., et al. 2005, ApJ, 621, 104

Turner, T. J., \& Pounds, K. A. 1989, MNRAS, 240, 833

Veilleux, S., \& Osterbrock, D. E. 1987, ApJS, 63, 295

Weingartner, J. C., \& Murray, N. 2002, ApJ, 580, 88

Worsley, M. A., Fabian, A. C., Alexander, D. M., et al. 2005, AIP Conf. Proc., 801,51 
O. Garcet et al.: The XMM large scale structure survey: optical vs. X-ray classifications of AGN, Online Material p 1

\section{Online Material}


O. Garcet et al.: The XMM large scale structure survey: optical vs. X-ray classifications of AGN, Online Material p 2

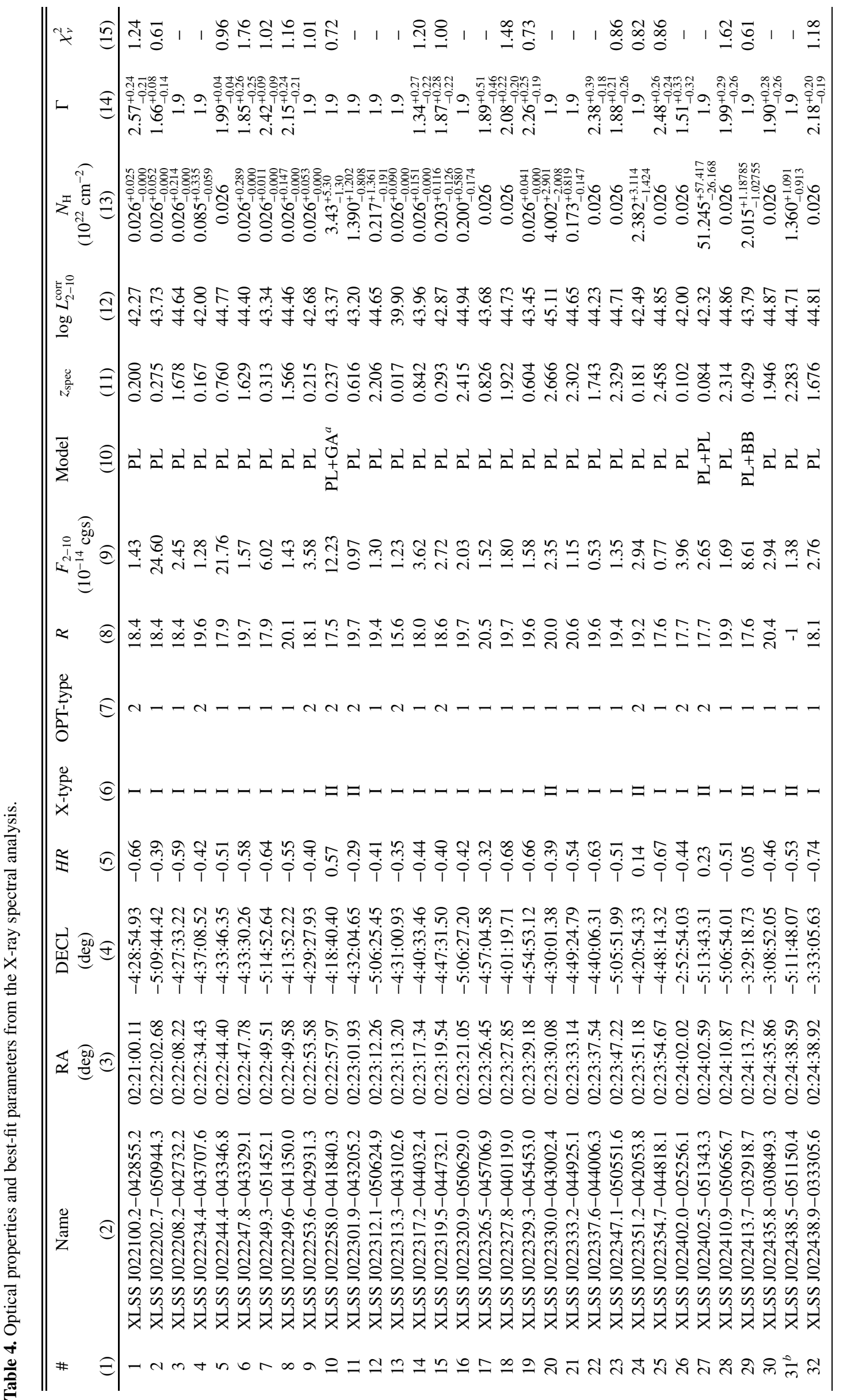


O. Garcet et al.: The XMM large scale structure survey: optical vs. X-ray classifications of AGN, Online Material p 3

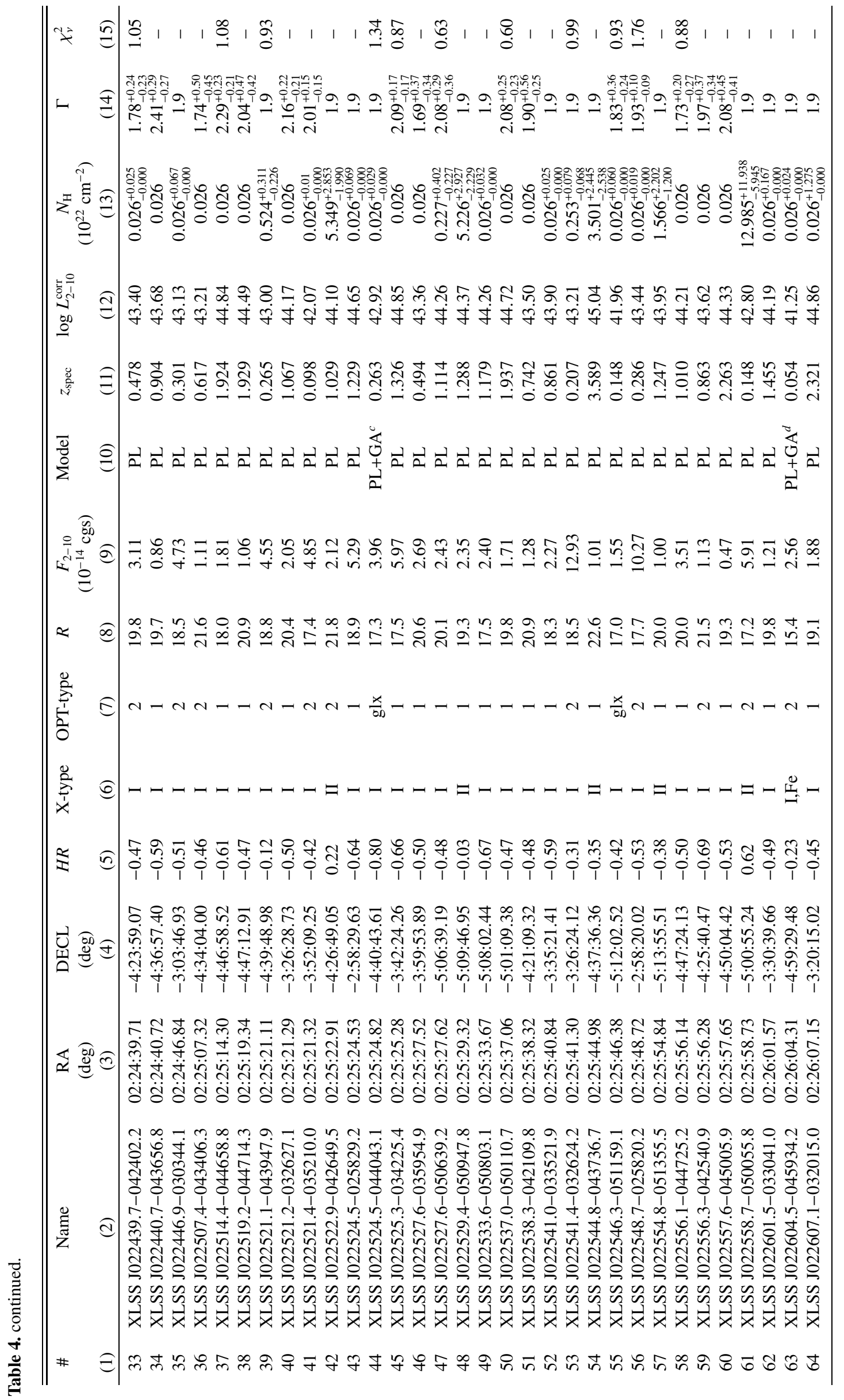


O. Garcet et al.: The XMM large scale structure survey: optical vs. X-ray classifications of AGN, Online Material p 4

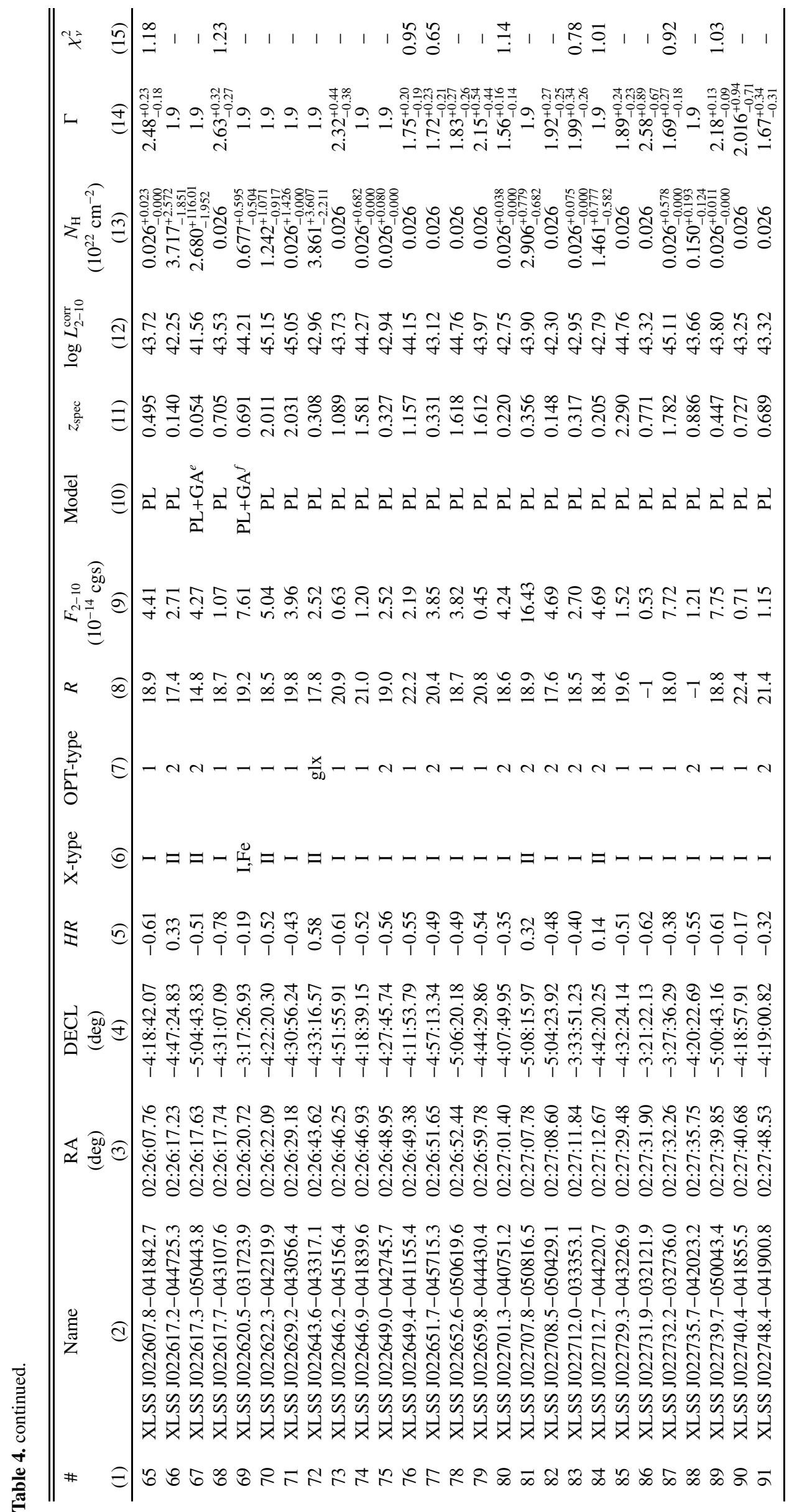


O. Garcet et al.: The XMM large scale structure survey: optical vs. X-ray classifications of AGN, Online Material p 5

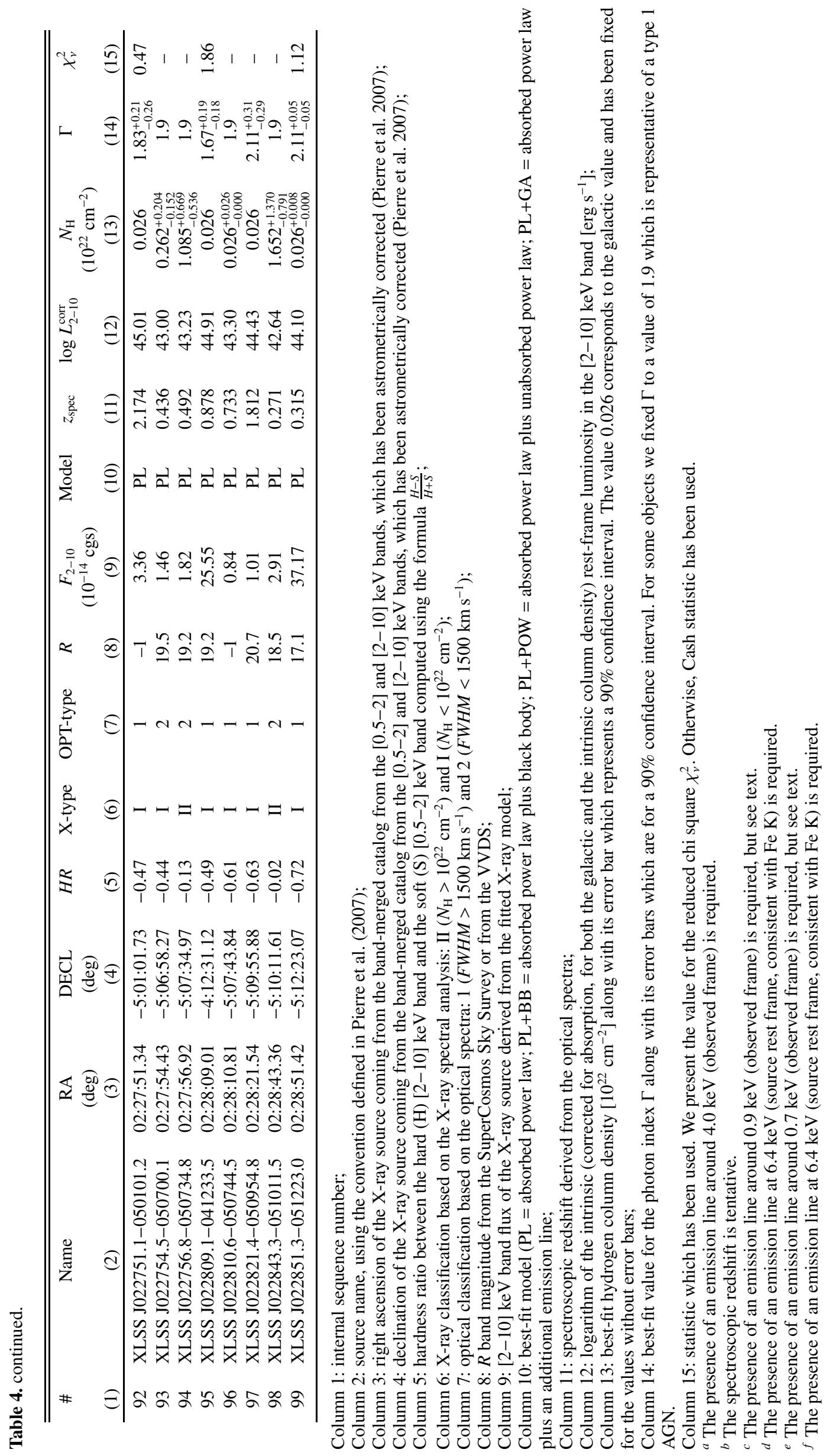


O. Garcet et al.: The XMM large scale structure survey: optical vs. X-ray classifications of AGN, Online Material p 6

\section{Appendix A: Illustrating figures about selection effects in our sample}

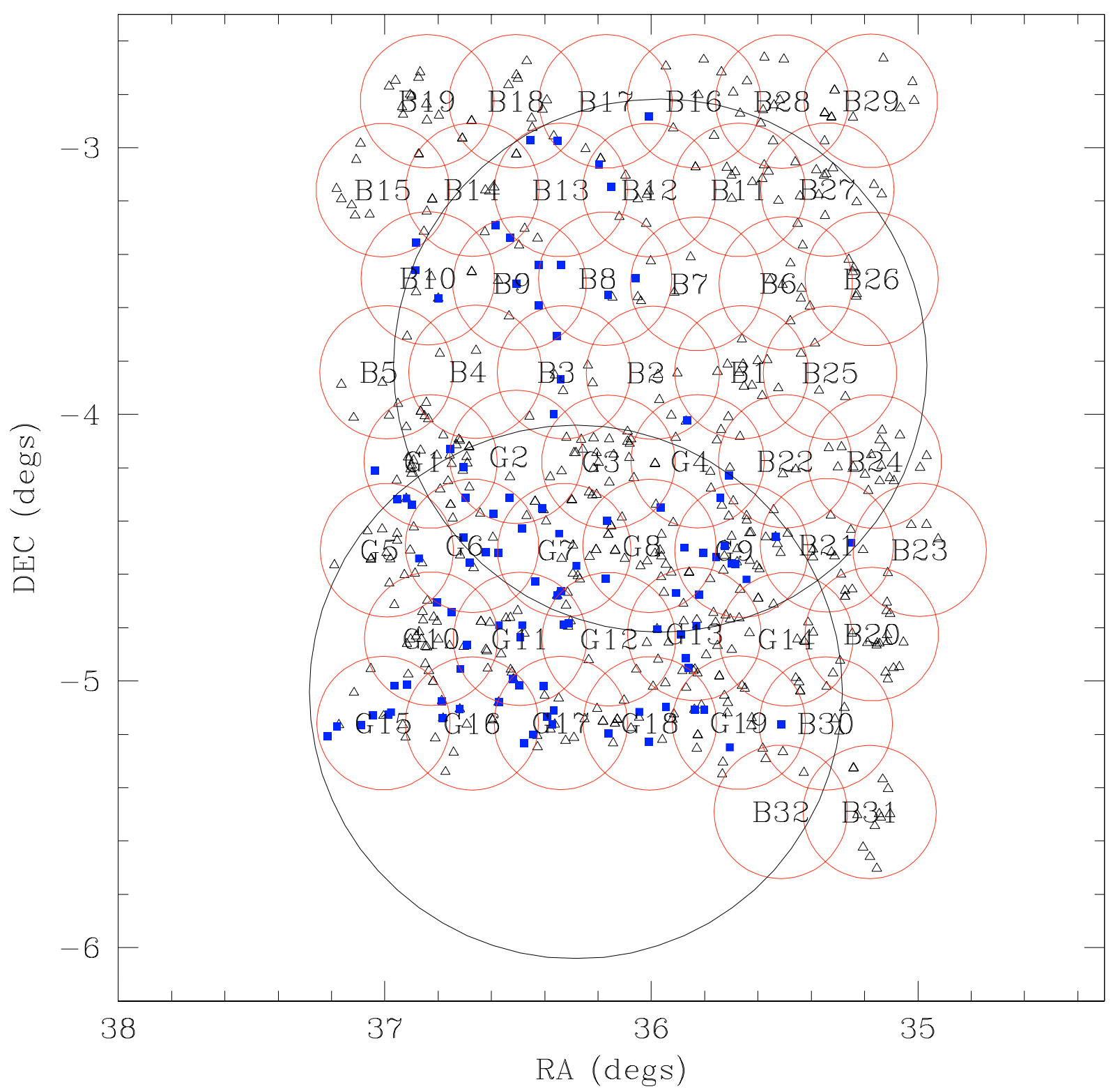

Fig. A.1. The layout of the $51 \mathrm{X}$-ray pointings of the XMM-LSS survey is such that most adjacent pointings overlap beyond 10 arcmin from their respective optical axis centers. The filled squares correspond to the 99 spectroscopically identified X-ray sources for this analysis, while the empty triangles correspond to the remaining 513 X-ray sources. Finally, the two large overlapping circles correspond to the two $2 \mathrm{dF}$ fields used in the spectral identification process. 
O. Garcet et al.: The XMM large scale structure survey: optical vs. X-ray classifications of AGN, Online Material p 7
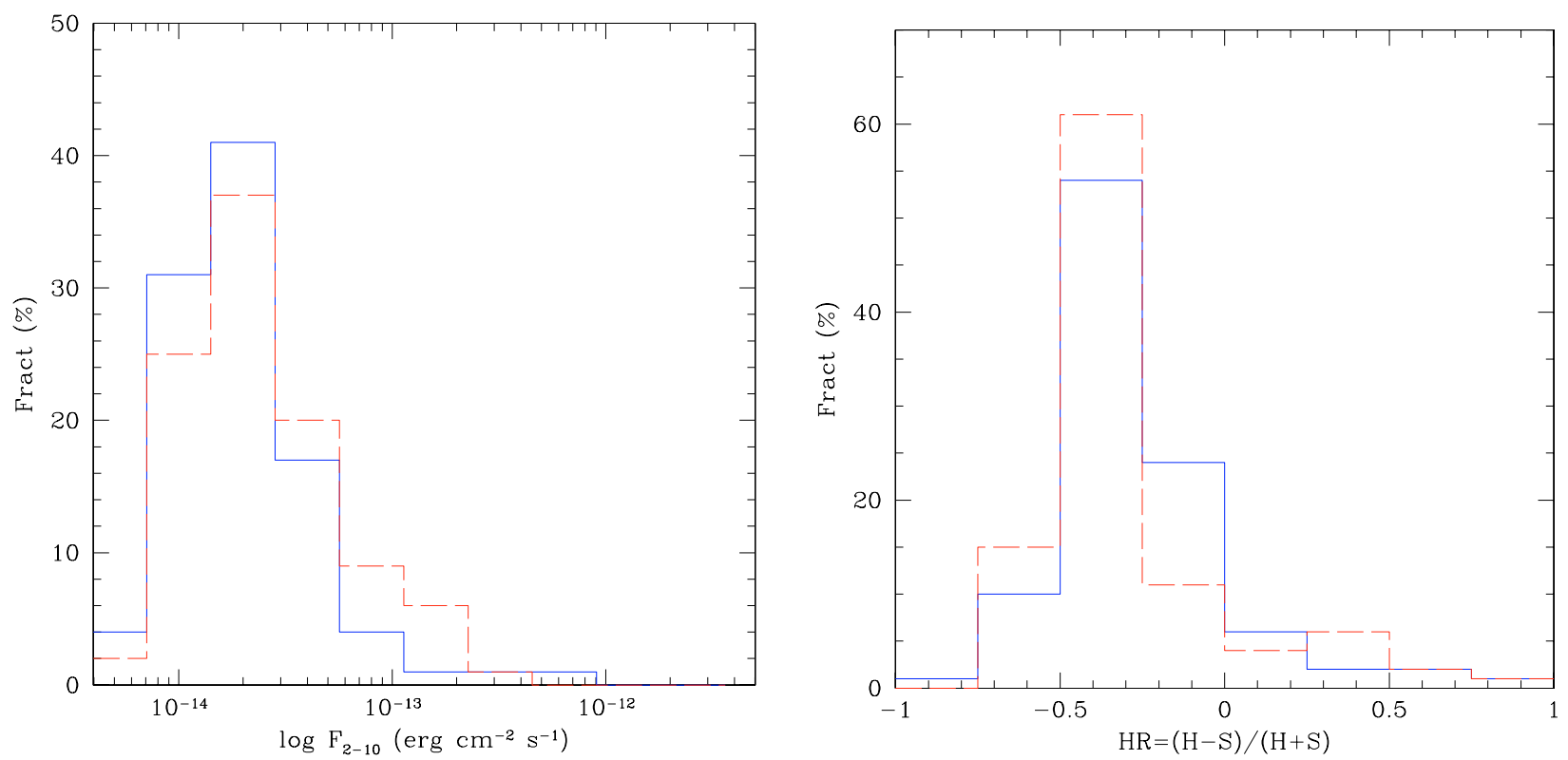

Fig. A.2. Left panel: normalized 2-10 keV band flux distribution for the 513 still optically unidentified X-ray sources (solid line) compared to the distribution of the sample of 99 spectroscopically selected X-ray point like sources (long dashed line). Right panel: normalized hardness ratio distribution for the 513 still optically unidentified X-ray sources (solid line) compared to that of the sample of 99 optically selected X-ray point like sources (long dashed line).
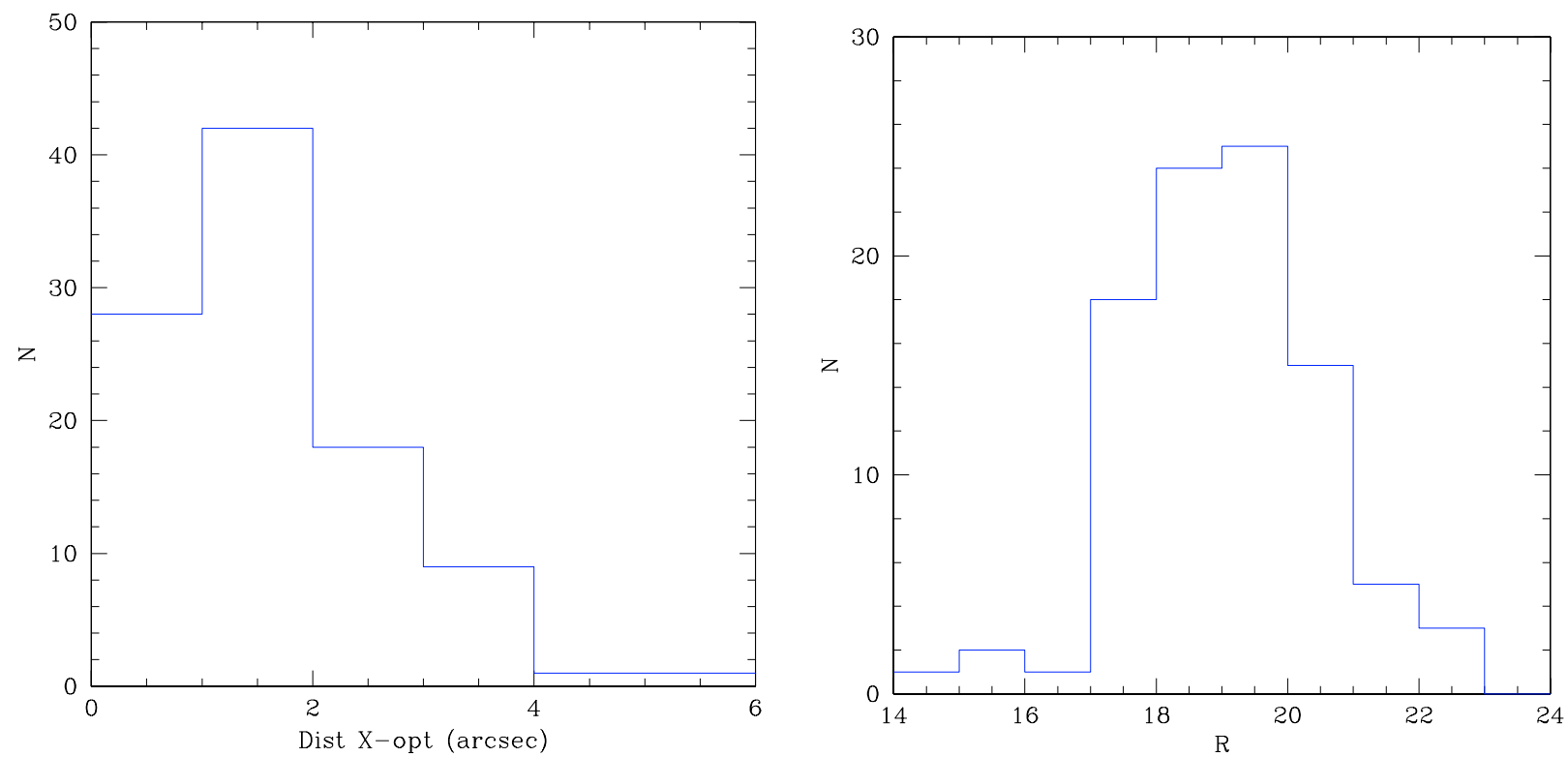

Fig. A.3. Left panel: histogram of the distribution of the separation between each X-ray source and its optical counterpart, for the 99 spectroscopically identified X-ray sources. Right panel: $R$ band magnitude distribution of the 94 optical counterparts of our sample of X-ray sources for which an $R$ band magnitude is available. For most of the objects, the magnitude is red magnitude from UKST plate scans, while the others are $R$ magnitudes from the VVDS. 